\title{
Gestational Diabetes Mellitus in Europe: A Systematic Review and Meta-Analysis of Prevalence Studies
}

\section{OPEN ACCESS}

Edited by:

Pradeep Kumar,

University of the Witwatersrand,

South Africa

Reviewed by:

Ewa Wender-Ozegowska,

Poznan University of Medical

Sciences, Poland

Dan Quansah,

Lausanne University Hospital,

Switzerland

${ }^{*}$ Correspondence:

Rami H. Al-Rifal

rrifai@uaeu.ac.ae

Specialty section: This article was submitted to Clinical Diabetes,

a section of the journal

Frontiers in Endocrinology

Received: 21 April 2021 Accepted: 17 November 2021 Published: 09 December 2021

Citation:

Paulo MS, Abdo NM, Bettencourt-Silva $R$ and Al-Rifai $R H$ (2021) Gestational Diabetes Mellitus in Europe: A Systematic Review and Meta-Analysis of Prevalence Studies.

Front. Endocrinol. 12:691033. doi: 10.3389/fendo.2021.691033

\author{
Marilia Silva Paulo ${ }^{1}$, Noor Motea Abdo ${ }^{1}$, Rita Bettencourt-Silva ${ }^{2,3}$ and Rami H. Al-Rifai ${ }^{1 *}$ \\ 1 Institute of Public Health, College of Medicine and Health Sciences, United Arab Emirates University, \\ Al Ain, United Arab Emirates, ${ }^{2}$ Department of Endocrinology and Nutrition, Unidade Local de Saúde do Alto \\ Minho, Viana do Castelo, Portugal, ${ }^{3}$ Department of Endocrinology, Hospital Lusíadas Porto, Porto, Portugal
}

Background: Gestational Diabetes Mellitus (GDM) is defined as the type of hyperglycemia diagnosed for the first-time during pregnancy, presenting with intermediate glucose levels between normal levels for pregnancy and glucose levels diagnostic of diabetes in the non-pregnant state. We aimed to systematically review and meta-analyze studies of prevalence of GDM in European countries at regional and subregional levels, according to age, trimester, body weight, and GDM diagnostic criteria.

Methods: Systematic search was conducted in five databases to retrieve studies from 2014 to 2019 reporting the prevalence of GDM in Europe. Two authors have independently screened titles and abstracts and full text according to eligibility using Covidence software. A random-effects model was used to quantify weighted GDM prevalence estimates. The National Heart, Lung, and Blood Institute criteria was used to assess the risk of bias.

Results: From the searched databases, 133 research reports were deemed eligible and included in the meta-analysis. The research reports yielded 254 GDM-prevalence studies that tested 15,572,847 pregnant women between 2014 and 2019. The 133 research reports were from 24 countries in Northern Europe (44.4\%), Southern Europe (27.1\%), Western Europe (24.1\%), and Eastern Europe (4.5\%). The overall weighted GDM prevalence in the 24 European countries was estimated at 10.9\% (95\% Cl: 10.0-11.8, $\left.P^{2}: 100 \%\right)$. The weighted GDM prevalence was highest in the Eastern Europe (31.5\%, 95\% Cl: 19.8-44.6, $I^{2}:$ 98.9\%), followed by in Southern Europe (12.3\%, 95\% Cl: 10.9-13.9, $I^{2}$ :

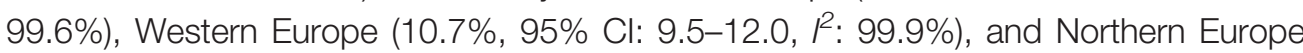
(8.9\%, 95\% Cl: 7.9-10.0, $I^{2}:$ 100). GDM prevalence was 2.14-fold increased in pregnant women with maternal age $\geq 30$ years (versus $15-29$ years old), 1.47-fold if the diagnosis was made in the third trimester (versus second trimester), and 6.79- fold in obese and 2.29-fold in overweight women (versus normal weight).

Conclusions: In Europe, GDM is significant in pregnant women, around 11\%, with the highest prevalence in pregnant women of Eastern European countries (31.5\%). Findings have implications to guide vigilant public health awareness campaigns about the risk factors associated with developing GDM.

Systematic Review Registration: PROSPERO [https://www.crd.york.ac.uk/ PROSPERO/], identifier CRD42020161857.

Keywords: diabetes mellitus, Europe, Gestational Diabetes Mellitus, GDM, systematic review, meta-analysis, pregnancy complications, pregnancy hyperglycemia 


\section{INTRODUCTION}

Hyperglycemia in pregnancy affects about one in every six pregnancies worldwide (1). Gestational Diabetes Mellitus (GDM) is defined as the type of hyperglycemia diagnosed for the first time during pregnancy $(2,3)$. This has been the widely used definition of GDM for many years, but it presents limitations in terms of the non-possible verification of the preexisting hyperglycemia (4). Hyperglycemia universal routine screening is not available for women at childbearing age before conception or in the first semester, so although GDM can take place at any time during pregnancy, it is more frequently diagnosed after the $24^{\text {th }}$ week of gestation $(1,4)$.

GDM is highly associated with obesity. Obesity is a growing major public health problem worldwide (5). In 2016, the estimated age-standardized prevalence of obesity and overweight among adult women of the European Region was $24.5 \%$ and $54.3 \%$, respectively (6). This prevalence is expected to continue rising in the next years $(7,8)$. Being overweight (body mass index [BMI] 25.0-29.9 $\mathrm{kg} / \mathrm{m}^{2}$ ) or obese $\left(\mathrm{BMI} \geq 30.0 \mathrm{~kg} / \mathrm{m}^{2}\right)$ is the most important modifiable risk factor for GDM. The risk is up to 5-fold higher in morbidly obese women, when compared to women with normal body weight (9). Other modifiable risk factors for GDM comprise unhealthy dietary factors, physical inactivity, and cigarette smoking (10). Moreover, the gradual increase in the mean age at childbearing of women in Europe (from 28.8 years in 2013 to 29.3 years in 2018) has an important role in the prevalence of GDM, given that advanced maternal age is a well-known risk factor for GDM (11). The chances of developing GDM increment with previous history of GDM, macrosomia, excessive gestational weight gain, spontaneous abortion, fetal anomalies, preeclampsia, fetal demise, neonatal hypoglycemia, hyperbilirubinemia, and neonatal respiratory distress syndrome family history of type 2 diabetes mellitus (T2DM), polycystic ovary syndrome, parity, non-white ancestry also increment $(10,12)$.

GDM has potentially serious short- and long-term consequences. The condition is associated with various adverse maternal, fetal, and perinatal outcomes, including but not limited to, preeclampsia, preterm delivery, cesarean section delivery, large for gestational age (LGA) newborns, neonatal hypoglycemia, and Neonatal Intensive Care Unit admission (13). The Hyperglycemia and Adverse Pregnancy Outcomes (HAPO) study reported a continuous association between maternal glucose levels and increased frequency of adverse outcomes, however, there was no obvious threshold at which risk increased (13). Furthermore, the gestational programming and intrauterine fetal exposure to

Abbreviations: ADA, American Diabetes Association; BMI, body mass index; CI, Confidence interval; DM, diabetes mellitus; EVOO, extra virgin olive oil; GDDD, Deutsche Gesellschaft fur gynakologie und Geburtshilfe; GDM, Gestational diabetes mellitus; HAPO, Hyperglycemia and Adverse Pregnancy Outcomes; HIV, Human Immunodeficiency Virus; IADPSG, International Association of the Diabetes and Pregnancy Study Groups; IDF, International Diabetes Federation; NICE, National Institute for Health and Care Excellence; OGTT, oral glucose tolerance test; PDA, Polish Diabetes Association; PECO, Population, Exposure, Comparator and Outcome; PRISMA, Preferred Reporting Items for Systematic Review and Meta-Analysis; RoB, risk of bias; T2DM, type 2 diabetes mellitus; UK, United Kingdom; UN, United Nations; WHO, World Health Organization. hyperglycemia is an independent risk factor for obesity, hypertension and T2DM in the offspring $(14,15)$. GDM may play a crucial role in increasing the prevalence of T2DM in women. In the European Region, about $9.6 \%$ of women $\geq 25$ years old have diabetes (16). A meta-analysis reported a 7-fold increased risk of T2DM in women with GDM compared with those without GDM (17).

Comparing data on GDM is a challenge since there is a lack of universally accepted screening standards and diagnostic criteria. Diagnostic criteria have changed over time and remain controversial, but there has been a move towards the adoption of the International Association of Diabetes in Pregnancy Study Groups (IADPSG) recommendations (18-20). Using the systematic review and meta-analysis approach to understand the regional, sub-regional, and national prevalence of GDM will help the introduction of effective public health measures and enable highlighting the gaps in evidence, following the Guidelines for Accurate and Transparent Health Estimates Reporting (GATHER) (21).

The previously published meta-analysis on the GDM prevalence in Europe was limited to only developed countries in Europe excluding immigrants who did not originate from those developed countries (22). Also, the same meta-analysis was limited to only women tested for GDM in their second or third trimesters (22). To overcome these limitations and provide a more comprehensive and informative assessment on the GDM prevalence in Europe, the present systematic review included all countries in the European region according to the definition of the United Nations (UN) geoscheme and regardless of the original of the included pregnant women. In the present review, the literature search covers a wider range of countries (51 countries) in the European continent regardless of the development status, the origin of the study population, and pregnancy trimester. Moreover, our meta-analyses considered extracting, whenever possible, stratified estimates of the GDM rather than using the overall prevalence reported in the primary studies following a prioritized one-stratification scheme. Indeed, pooling stratified estimates would provide more precise findings on the national, sub-regional, and regional prevalence of GDM. As such, this systematic review and meta-analysis method quantifies the weighted prevalence of GDM in Europe, at regional, subregional, and national levels, between 2014 and 2019, according to and regardless of the maternal age, trimester, maternal weight, and GDM diagnostic criteria. It is believed that this study of the 51 countries of the European region regardless of their development will complement the scientific literature, providing more insights into the prevalence of GDM at the subregional level as countries within each subregion in the European continent might have not the same development status interpreted as a limitation in the previous systematic review (22).

\section{METHODS}

\section{Protocol and Registration}

We have developed and registered our protocol on PROSPERO (registration number: CRD42020161857). This systematic review 
and meta-analysis follows the Preferred Reporting Items for Systematic Review and Meta-Analysis (PRISMA) statement (23). The PRISMA checklist is provided elsewhere (see Supplementary Table S1).

This systematic review and meta-analysis from prevalence studies in Europe is part of a major study that aims to estimate the prevalence of GDM in different regions in the world. From the same project, the first systematic review and meta-analysis providing findings on the prevalence of GDM in the Middle East and North Africa region has been already completed and submitted for a peer-reviewed journal (24).

\section{Eligibility Criteria}

The search strategy was limited to English language publications between January 2006 and December 2019 and defined in accordance with our population, exposure, comparator, and outcome (PECO) criteria. The population included in this study were all pregnant women tested for GDM during their pregnancy, living in the European region according to the definition of the United Nations (UN) geoscheme (25). All included studies had at least ten pregnant women tested for GDM and reported the prevalence of GDM for their sample or have reported data that allowed us to calculate the GDM prevalence, regardless of the age, trimester, pregnancy status, or GDM ascertainment methodology. However, due to the high number of studies retrieved from databases, we restricted the inclusion criteria to only include studies published between 2014 and 2019.

All studies reporting prevalence estimates on GDM were considered eligible. For this specific systematic review and meta-analysis focusing on the European region, we have excluded studies from the other regions of the globe and studies using unclear GDM diagnostic criteria, unless studies from medical records. These decisions made by the research team were due to the high volume of eligible studies and to produce less potentially biased and more precise estimates on the GDM prevalence.

\section{Information Sources and Search}

A specific search strategy was developed by the principal investigators and a medical librarian expert. The initial search was developed on PubMed-MEDLINE using varied Medical Subject Headings (MeSH) and free-text terms and then translated into EMBASE, Scopus, Web of Sciences, and Cochrane Library, comprising five electronic databases (Supplementary Table S2).

\section{Study Selection}

We have used the Covidence software $(26,27)$ to perform study selection. All citations identified by our search strategy were uploaded into Covidence where duplicates were automatically removed. Two reviewers independently screened the studies for titles and abstracts and subsequently identified potential eligible full-text articles. Conflicts and discrepancies that emerged during the two stages of screening were solved by a third reviewer. The reference lists of eligible studies were also screened to identify additional studies that might have been missed.

\section{Data Abstraction Process and Data Items}

The data we have extracted include the study ID, article type, publication year, journal, country, city, study design, data collection period, population, sample size, sampling strategy, age, pregnancy trimester when GDM was tested, GDM criteria used for diagnosis ascertainment, strata used on the population of the study, the prevalence of GDM in the study sample and by strata whenever available. Furthermore, in research reports presenting more stratified GDM prevalence and at least ten tested subjects per strata, we have extracted the stratified prevalence of GDM following a priority list to avoid double counting: comorbidity, parity, age, pre-gestational BMI, ethnicity, year, placental location, nationality, and occupation. Where there was no stratification on the prevalence of GDM, the overall prevalence was extracted. All relevant data were introduced into a predesigned Excel sheet using string codes and numerical variables. We considered a research report a single publication that might contain data from several studies (each one on a specific population group). In reports where the main study design does not report a clear prevalence, we have extracted the original study design of the report and we have calculated the prevalence of GDM accordingly. In reports where the GDM was ascertained using more than one criterion, the most sensitive and reliable assessment (e.g., fasting glucose blood test $v s$. self-reported) was considered as well as the most recent criteria (e.g., The American Diabetes Association ADA 2010 vs. ADA 2006).

\section{Summary Measures and Synthesis of Results}

To estimate the weighted pooled prevalence of GDM and the corresponding 95\% confidence interval (CI), we performed meta-analyses of the extracted data. The Freeman-Tukey double arcsine transformation method was applied to stabilize the variances of the prevalence measures (28). The inverse variance method was used to weight the estimated pooled prevalence measures (29). Dersimonian-Laird random-effects model was used to estimate the overall pooled GDM prevalence (30). Cochran's Q statistic and the inconsistency index I-squared $\left(I^{2}\right)$, were calculated to measure heterogeneity. Along with the pooled estimates, ranges and median were also reported to describe the dispersion of the GDM prevalence measures reported in the literature. The prediction interval, which estimates the $95 \%$ interval in which the true prevalence of GDM in a new study will lie, was also quantified and reported (31).

The overall, country-level and sub-regional levels [Eastern Europe, Northern Europe, Western Europe, and Southern Europe (25)] pooled GDM prevalence was estimated. Moreover, within each sub-European region, the pooled GDM prevalence estimates were generated overall and based on age $(<30, \geq 30$, or unclear age group), pregnancy trimester (first, second, third, or unclear trimester), BMI (normal, overweight, obsess, or unclear BMI), and GDM ascertainment criteria. The provision of pooled estimates regardless of the ascertainment guidelines was justified by the fact that the women were defined 
and treated as GDM patients following each specific ascertainment guideline. We conducted a synthesis of results including the above-described meta-analysis also comprises a description of the main findings relevant to the study.

\section{Risk of Bias (RoB)}

To test the robustness of the implemented methodology, quality of evidence criteria was also used GDM ascertainment method, sampling methodology, and precision of the estimate. The risk of bias (RoB) tool was performed for each research report and not for individual studies, using the six-quality items adapted from the National Heart, Lung, and Blood Institute (NIH) criteria (32). From the 14 items of the NIH RoB tool we used research question/objective, studied population, participation rate, recruitment, sample size justification, and outcome measures and assessment. Reports were considered to have "high" precision if at least 100 women were tested for GDM. We computed the overall proportion of research reports with potentially low RoB across each of these nine quality criteria and the proportion (out of nine) of quality items with a potentially low RoB for each of the included research reports.

\section{Publication Bias}

The small-study effect on the pooled GDM prevalence estimates was explored through plotting the funnel plot. In the funnel plot, each GDM prevalence measure was plotted against its standard error. The asymmetry of the funnel plot was tested using Egger's test (33).

Analyses were performed using the metaprop (34) and metareg packages in Stata/SE v15 (35).

\section{RESULTS}

\section{Study Selection}

After de-duplication, 15,933 records were screened and 547 fulltext research reports critically assessed for eligibility, 133 research reports were deemed eligible and included in the meta-analysis (Figure 1).

\section{Study Characteristics}

The 133 research reports related to 24 countries in Europe and tested a total of 15,572,847 pregnant women for GDM and yielded 254 GDM prevalence studies. The majority of the research reports were reported from Northern Europe (59/ 133), followed by Southern Europe (36/133), Western Europe (32/133), and Eastern Europe (6/133). Across the four UN geoscheme sub-regions (25) the most studied countries were Italy (21 reports) and the United Kingdom (14 reports). Tables 1-4 summarize basic characteristics of the included research articles in the four European sub-regions.

\section{Eastern Europe}

From the Eastern Europe countries (Belarus, Bulgaria, Czech Republic, Hungary, Poland, Republic of Moldova, Romania, Russian Federation, Slovakia, and Ukraine), our search has just captured six reports that tested a total of 12,122 pregnant women for GDM from Hungary (two reports), Poland (three reports), and Republic of Macedonia (one report). In two out of the eight GDM prevalence studies reported in these three countries, GDM ascertainment was based on the Polish Gynecological Society Guidelines (Table 1).

\section{Northern Europe}

From Northern Europe sub-region (Denmark, Estonia, Finland, Iceland, Ireland, Latvia, Lithuania, Norway, Sweden, and United Kingdom), there were 59 reports presenting estimates on GDM prevalence. None of those reports were from Estonia or Latvia. Seven reports reporting 17 GDM prevalence studies were from Denmark, 10 reports with 22 GDM prevalence studies were from Finland, one report with three GDM prevalence studies were from Iceland, seven reports with 10 GDM prevalence studies were from Ireland, two reports with three GDM prevalence studies were from Lithuania, nine reports with 19 GDM prevalence were studies from Norway, nine reports with 20 GDM prevalence were studies from Sweden and 14 reports with 28 GDM prevalence studies were from the United Kingdom. In the 122 GDM prevalence studies that tested a total of $10,278,921$ pregnant women reported in the Northern European countries, the IADPSG (in 15 out of 122 studies) followed by the WHO 2013 (in 14 out of 122 studies) were the most common used GDM diagnostic (Table 2).

\section{Western Europe}

From Western Europe sub-region (Austria, Belgium, France, Germany, Liechtenstein, Luxembourg, Monaco, Netherlands, and Switzerland). In this sub-region, the majority of the 32 research reports were in France $(34.4 \%)$ followed by Germany (18.8\%), Austria (15.6\%), and Switzerland (15.6\%). Our study did not find any prevalence studies on GDM from three countries (Liechtenstein, Luxembourg, and Monaco) in this sub-region reported between 2014 and 2019. In the 55 GDM prevalence studies that tested a total of 4,212,723 pregnant women in the Western European countries, the IADPSG (in 14 studies) was the most commonly used GDM diagnostic (Table 3).

\section{Southern Europe}

From Southern Europe sub-region (Albania, Andorra, Bosnia and Herzegovina, Croatia, Greece, Italy, Malta, Montenegro, North Macedonia, Portugal, San Marino, Serbia, Slovenia, and Spain), there were 36 research reports, of which, the majority were from Italy (58.3\%) followed by $19.4 \%$ were from Spain. Between 2014 and 2019, there were no prevalence studies on GDM from Albania, Andorra, Bosnia and Herzegovina, Montenegro, North Macedonia, Portugal, San Marino, and Serbia. In the 69 GDM prevalence studies that tested a total of $1,069,081$ pregnant women, the IADPSG was the most common GDM ascertainment criteria used (30.4\%) (Table 4).

\section{Weighted GDM Prevalence}

In the $15,572,847$ pregnant women tested for GDM the weighted GDM prevalence estimated was 10.9\% (95\% CI: $10.0-11.8 \%, I^{2}$, $100 \%$ ) in the 24 countries out of a total of 48 countries in Europe. Of the tested pregnant women, $76.6 \%$ were from three countries: 


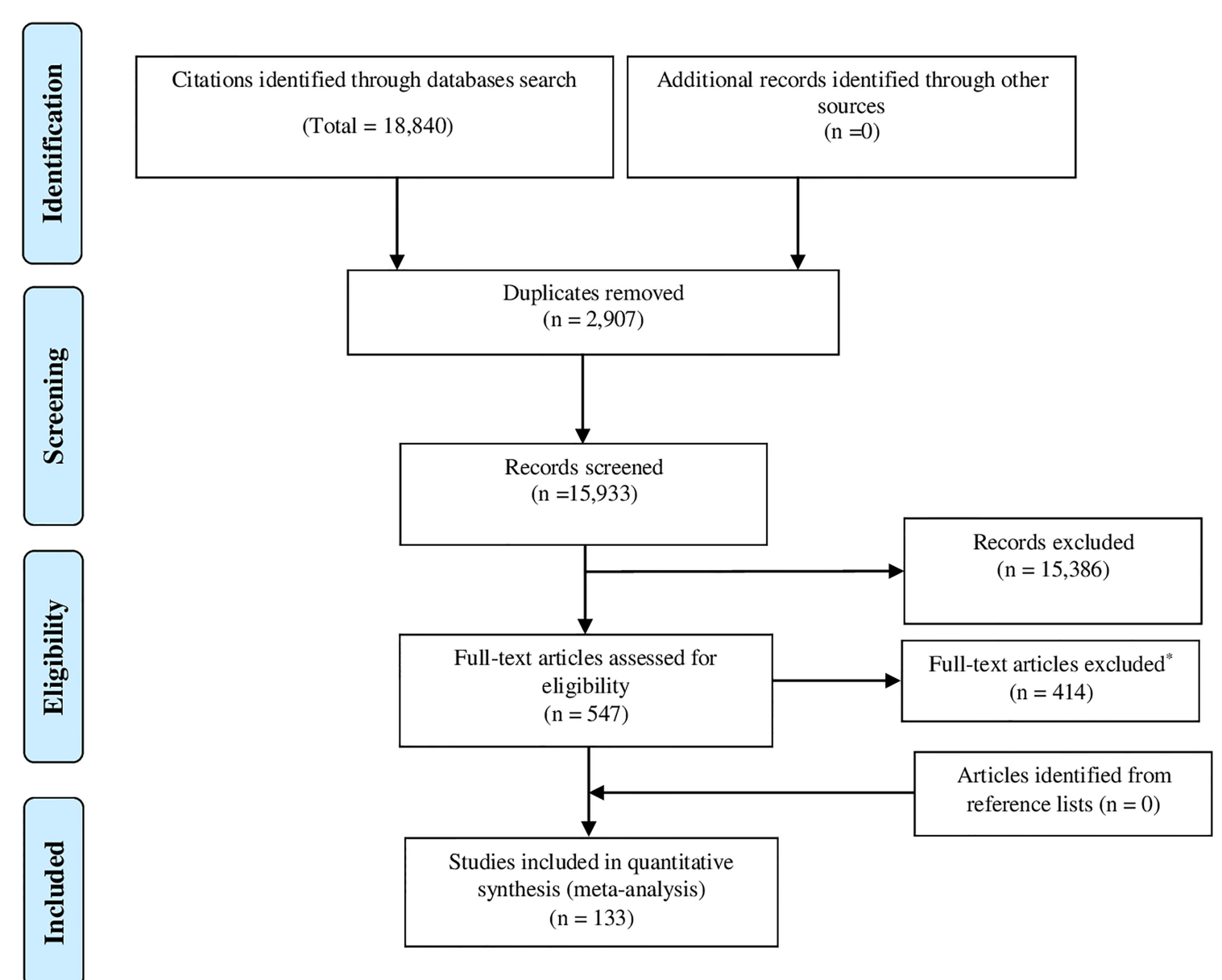

FIGURE 1 | PRISMA flowchart. • Reasons for full-text exclusion: 214 GDM or DM total population 78 Wrong setting 29 Not in Europe 24 GDM Prevalence was incalculable 23 reported an unclear ascertainment of GDM criteria (I report containing information front Albania, 3 from Denmark, 2 from Finland, 2 from Ireland, 5 front Italy, I from Netherlands, 2 f:om Poland, 2 from Portugal, 2 from Spain, and 3 from United Kingdom). 15 reports have duplicate data [l from Croatia (30), I from France (31), I from Italy (32), 2 from Netherlands (33,34),6 from Norway (35-40), and 4 from United Kingdom (41-44)], and only the report that first published the study data was used. 9 Conference abstract with not enough information 8 Case-control (GDM vs. non-GDM) 7 Duplicates 6 Wrong patient population 1 Year of GDM diagnosis is UNCLEAR (Not mentioned).

TABLE 1 | Baseline studies characteristics from Eastern Europe.

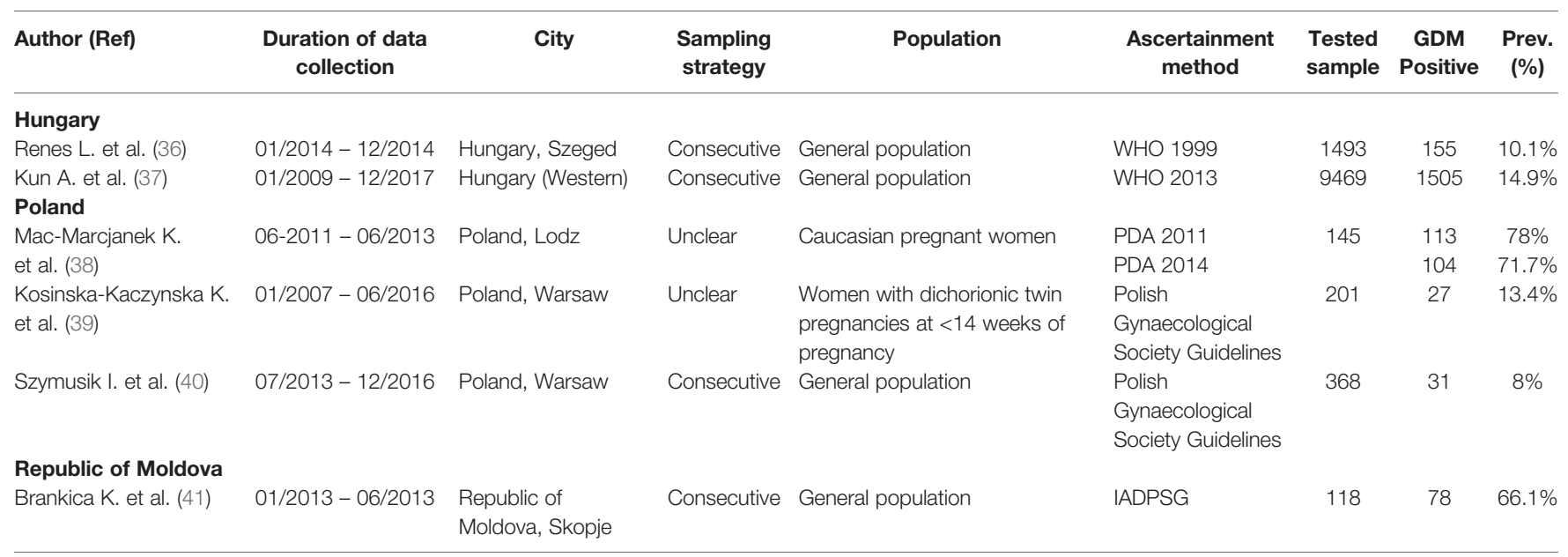

IADPSG, International Association of Diabetes in Pregnancy Studies Group; PDA, Polish Diabetes Association; WHO, World Health Organization. 
TABLE 2 | Baseline studies characteristics from Northern Europe.

\begin{tabular}{|c|c|c|c|c|c|c|c|c|}
\hline Author (Ref) & $\begin{array}{l}\text { Duration of data } \\
\text { collection }\end{array}$ & City & $\begin{array}{l}\text { Sampling } \\
\text { strategy }\end{array}$ & Population & $\begin{array}{l}\text { Ascertainment } \\
\text { method }\end{array}$ & $\begin{array}{l}\text { Tested } \\
\text { sample }\end{array}$ & $\begin{array}{c}\text { GDM } \\
\text { Positive }\end{array}$ & $\begin{array}{l}\text { Prev. } \\
(\%)\end{array}$ \\
\hline \multicolumn{9}{|l|}{ Denmark } \\
\hline Bonnesen B. et al. (42) & 01/2009-12/2010 & Denmark, Hvidovre & Consecutive & $\begin{array}{l}\text { Primiparous women } \\
\text { with a spontaneous } \\
\text { singleton pregnancy }\end{array}$ & Medical Records & 3,440 & 43 & $1.3 \%$ \\
\hline Medek H. et al. (43) & 05/2012 - 10/2013 & Denmark, Reykjavik & Consecutive & General population & IADPSG & 117 & 22 & $12.4 \%$ \\
\hline Holst S. et al. (44) & $01 / 2006-12 / 2010$ & Denmark, National & $\begin{array}{l}\text { Whole } \\
\text { population }\end{array}$ & $\begin{array}{l}\text { Women with singleton } \\
\text { pregnancies }\end{array}$ & Medical Records & 264539 & 5781 & $2.2 \%$ \\
\hline Jeppesen C. et al. (45) & $01 / 2012-12 / 2012$ & Denmark, National & $\begin{array}{l}\text { Whole } \\
\text { population }\end{array}$ & $\begin{array}{l}\text { Women aged 15-49 } \\
\text { years old }\end{array}$ & Medical Records & 56894 & 1721 & $3.0 \%$ \\
\hline Mclntyre HD. et al. (10) & $01 / 2010-12 / 2012$ & Denmark, National & $\begin{array}{l}\text { Whole } \\
\text { population }\end{array}$ & General population & WHO 2013 & 1516 & 620 & $40.1 \%$ \\
\hline \multirow[t]{4}{*}{ Hamann CR. et al. (46) } & 01/1997-12/2014 & Denmark, National & Whole & General population & Medical Records & 104410 & 1914 & $1.8 \%$ \\
\hline & & & population & $\begin{array}{l}\text { Women with atopic } \\
\text { dermatitis any time } \\
\text { prior to birth }\end{array}$ & & 10441 & 175 & $1.7 \%$ \\
\hline & & & & $\begin{array}{l}\text { Women with atopic } \\
\text { dermatitis } 24 \text { months } \\
\text { prior to birth }\end{array}$ & & 1064 & 15 & $1.4 \%$ \\
\hline & & & & $\begin{array}{l}\text { Women with atopic } \\
\text { dermatitis during } \\
\text { pregnancy }\end{array}$ & & 319 & 3 & $0.9 \%$ \\
\hline Strand-Horlm KM. et al. (47) & $01 / 2004-12 / 2012$ & Denmark, Aarhus & $\begin{array}{l}\text { Whole } \\
\text { population }\end{array}$ & $\begin{array}{l}\text { Women with singleton } \\
\text { pregnancies }\end{array}$ & WHO 2013 & 42571 & 928 & $2.2 \%$ \\
\hline \multicolumn{9}{|l|}{ Finland } \\
\hline Koivusalo SB. et al. (48) & $01 / 2008-12 / 2014$ & $\begin{array}{l}\text { Finland, } \\
\text { Lappeenranta }\end{array}$ & $\begin{array}{l}\text { Random } \\
\text { selection }\end{array}$ & $\begin{array}{l}\text { Women with a history } \\
\text { of GDM or pre- } \\
\text { pregnancy obesity }\end{array}$ & ADA 2007 & 269 & 47 & $17.4 \%$ \\
\hline Ellenberg A. et al. (49) & $\begin{array}{l}01 / 2006-12 / 2008 \\
01 / 2010-12 / 2012\end{array}$ & Finland, National & $\begin{array}{l}\text { Whole } \\
\text { population }\end{array}$ & $\begin{array}{l}\text { Women with singleton } \\
\text { pregnancies }\end{array}$ & Medical records & $\begin{array}{l}34460 \\
36331\end{array}$ & $\begin{array}{l}2522 \\
4128\end{array}$ & $\begin{array}{c}7.2 \% \\
11.3 \%\end{array}$ \\
\hline Koivunen S. et al. (50) & $\begin{array}{l}01 / 2006-12 / 2006 \\
01 / 2012-12 / 2010\end{array}$ & Finland, National & Consecutive & $\begin{array}{l}\text { Pregnant at } \\
\text { gestational age } \geq 22 \\
\text { weeks or a birthweight } \\
\geq 500 \mathrm{~g}\end{array}$ & $\begin{array}{l}\text { The Finnish } \\
\text { Current Care } \\
\text { guidelines }\end{array}$ & $\begin{array}{l}15682 \\
30365\end{array}$ & $\begin{array}{l}5179 \\
6679\end{array}$ & $\begin{array}{c}9.1 \% \\
11.3 \%\end{array}$ \\
\hline Meinilä J. et al. (51) & 01/2008 - 12/2014 & $\begin{array}{l}\text { Finland, Helsinki } \\
\text { Metropolitan area } \\
\text { and Lappeenranta }\end{array}$ & Unclear & $\begin{array}{l}\text { Women at high risk of } \\
\text { GDM due to obesity, } \\
\text { history of GDM, or } \\
\text { both }\end{array}$ & ADA 2008 & 251 & 46 & $18.3 \%$ \\
\hline Laine MK. et al. (52) & $01 / 2009-12 / 2015$ & Finland, Vantaa & $\begin{array}{l}\text { Whole } \\
\text { population }\end{array}$ & Primiparous women & $\begin{array}{l}\text { The Finnish } \\
\text { Current Care } \\
\text { guidelines }\end{array}$ & 7750 & 1281 & $16.5 \%$ \\
\hline Laine MK. et al. (53) & $01 / 2009-12 / 2015$ & Finland, Vantaa & $\begin{array}{l}\text { Whole } \\
\text { population }\end{array}$ & $\begin{array}{l}\text { Primiparous women } \\
\text { with height < } 159 \mathrm{~cm} \\
\text { Primiparous women } \\
\text { Primiparous women } \\
\text { with height between } \\
164-167 \mathrm{~cm}\end{array}$ & $\begin{array}{l}\text { The Finnish } \\
\text { Current Care } \\
\text { guidelines }\end{array}$ & $\begin{array}{l}689 \\
1221\end{array}$ & $\begin{array}{l}198 \\
243\end{array}$ & $\begin{array}{l}28.7 \% \\
19.9 \%\end{array}$ \\
\hline Girchenko P. et al. (54) & $01 / 2011-12 / 2012$ & Finland, National & $\begin{array}{l}\text { Whole } \\
\text { population }\end{array}$ & General population & Medical records & 2504 & 248 & $9.9 \%$ \\
\hline Kong L. et al. (55) & $01 / 2004-12 / 2014$ & Finland, National & $\begin{array}{l}\text { Whole } \\
\text { population }\end{array}$ & General population & Medical records & 649043 & 98568 & $15.2 \%$ \\
\hline Ellfolk M. et al. (56) & 01/1996-12/2016 & Finland, National & $\begin{array}{l}\text { Whole } \\
\text { population }\end{array}$ & $\begin{array}{l}\text { Women exposed to } \\
\text { antipsychotics }\end{array}$ & Medical records & 21125 & 3047 & $14.4 \%$ \\
\hline ljas H. et al. (57) & $01 / 2009-12 / 2009$ & Finland, National & $\begin{array}{l}\text { Whole } \\
\text { population }\end{array}$ & $\begin{array}{l}\text { Women with singleton } \\
\text { pregnancies }\end{array}$ & Medical records & 24555 & 5658 & $23.4 \%$ \\
\hline \multicolumn{9}{|l|}{ Iceland } \\
\hline Tryggvadottir EA. et al. (58) & 04/2012 - 10/2013 & Iceland, Reykjavik & Consecutive & $\begin{array}{l}\text { Non-smoking women } \\
\text { and without GDM risk } \\
\text { factors }\end{array}$ & WHO 2013 & 168 & 17 & $10.1 \%$ \\
\hline \multicolumn{9}{|l|}{ Ireland } \\
\hline Lindsay KL. et al. (59) & 03/2012 - 03/2013 & Ireland, Dublin & $\begin{array}{l}\text { Random } \\
\text { sampling }\end{array}$ & Obese women & $\begin{array}{l}\text { Carpenter and } \\
\text { Coustin }\end{array}$ & 138 & 6 & $4.3 \%$ \\
\hline
\end{tabular}


TABLE 2 | Continued

\begin{tabular}{|c|c|c|c|c|c|c|c|c|}
\hline Author (Ref) & $\begin{array}{l}\text { Duration of data } \\
\text { collection }\end{array}$ & City & $\begin{array}{l}\text { Sampling } \\
\text { strategy }\end{array}$ & Population & $\begin{array}{l}\text { Ascertainment } \\
\text { method }\end{array}$ & $\begin{array}{l}\text { Tested } \\
\text { sample }\end{array}$ & $\begin{array}{c}\text { GDM } \\
\text { Positive }\end{array}$ & $\begin{array}{c}\text { Prev. } \\
(\%)\end{array}$ \\
\hline Daly N. et al. (60) & 04/2014 - 08/2014 & Ireland, Dublin & Convenience & $\begin{array}{l}\text { Obese European } \\
\text { women }\end{array}$ & WHO 2013 & 24 & 16 & $66.7 \%$ \\
\hline Mone F. et al. (61) & $01 / 2011-09 / 2012$ & Ireland, Dublin & $\begin{array}{l}\text { Whole } \\
\text { population }\end{array}$ & General population & WHO 2013 & 7252 & 140 & $1.9 \%$ \\
\hline Moore R. et al. (62) & $2007-2013$ & Ireland, Dublin & Unclear & HIV women & $\begin{array}{l}\text { Carpenter and } \\
\text { Coustin }\end{array}$ & 142 & 3 & $2.1 \%$ \\
\hline O'Dea A. et al. (63) & $01 / 2013-12 / 2013$ & Ireland, Galway & Convenience & General population & IADPSG & 690 & 48 & $7.0 \%$ \\
\hline Farren N. et al. (64) & $01 / 2014-01 / 2016$ & Ireland, Dublin & Consecutive & $\begin{array}{l}\text { Women with family } \\
\text { history of DM }\end{array}$ & IADPSG & 240 & 40 & $16.6 \%$ \\
\hline \multirow[t]{2}{*}{ Daly N. et al. (65) } & $11 / 2013-04 / 2016$ & Ireland, Dublin & Consecutive & $\begin{array}{l}\text { Women with } \mathrm{BMI} \geq 30 \\
\text { that participated in the } \\
\text { intervention }\end{array}$ & IADPSG & 43 & 25 & $58.1 \%$ \\
\hline & & & & $\begin{array}{l}\text { Women with } \mathrm{BMI} \geq 30 \\
\text { that did not participate } \\
\text { in the intervention }\end{array}$ & & 43 & 21 & $48.8 \%$ \\
\hline \multicolumn{9}{|l|}{ Lithuania } \\
\hline \multirow[t]{2}{*}{ Ramoniene G. et al. (66) } & $01 / 2010-12 / 2010$ & Lithuania, Khaunas & Consecutive & $\begin{array}{l}\text { Obese women with } \\
\text { singletons }\end{array}$ & WHO 1999 & 140 & 33 & $23.6 \%$ \\
\hline & & & & $\begin{array}{l}\text { Normal weight women } \\
\text { with singletons }\end{array}$ & & 3107 & 160 & $5.1 \%$ \\
\hline Malakauskiene L. et al. (67) & $01 / 2005-12 / 2015$ & Lithuania, National & $\begin{array}{l}\text { Whole } \\
\text { population }\end{array}$ & $\begin{array}{l}\text { Pregnant after bariatric } \\
\text { surgery }\end{array}$ & Medical records & 130 & 3 & $2.31 \%$ \\
\hline \multicolumn{9}{|l|}{ Norway } \\
\hline Rasmussen S. et al. (68) & $01 / 2007$ - 12/2010 & Norway, National & $\begin{array}{l}\text { Whole } \\
\text { population }\end{array}$ & General population & Medical records & 77294 & 1086 & $1.4 \%$ \\
\hline Sommer C. et al. (69) & 05/2008 - 05/2010 & Norway, Oslo & Unclear & General population & IADPSG & 728 & 229 & $31.5 \%$ \\
\hline Helseth R. et al. (70) & 04/2007 - 06/2009 & $\begin{array}{l}\text { Norway, Trondheim, } \\
\text { and Stavanger }\end{array}$ & Unclear & $\begin{array}{l}\text { Nordic Caucasian } \\
\text { women }\end{array}$ & WHO 2013 & 687 & 42 & $6.1 \%$ \\
\hline Leirgul E. et al. (71) & $01 / 2006-12 / 2009$ & Norway, National & $\begin{array}{l}\text { Whole } \\
\text { population }\end{array}$ & General population & Medical records & 233303 & 3484 & $1.5 \%$ \\
\hline \multirow[t]{2}{*}{ Garnæs KK. et al. (72) } & $11 / 2012-03 / 2013$ & Norway, Trondheim & Unclear & $\begin{array}{l}\text { Women with } \mathrm{BMI} \geq 28 \\
\text { that participated in the } \\
\text { intervention }\end{array}$ & WHO 2013 & 46 & 8 & $18.2 \%$ \\
\hline & & & & $\begin{array}{l}\text { Women with } \mathrm{BMI} \geq 28 \\
\text { that did not participate } \\
\text { in the intervention }\end{array}$ & & 45 & 13 & $29.5 \%$ \\
\hline Sorbye LM. et al. (73) & $01 / 2006-12 / 2014$ & Norway, National & $\begin{array}{l}\text { Whole } \\
\text { population }\end{array}$ & $\begin{array}{l}\text { Women in their } \\
\text { second pregnancy }\end{array}$ & $\begin{array}{l}\text { Norwegian } \\
\text { Society of } \\
\text { Gynecology and } \\
\text { Obstetrics }\end{array}$ & 24198 & 439 & $1.8 \%$ \\
\hline Lehmann S. et al. (74) & $01 / 1967-12 / 2014$ & Norway, National & $\begin{array}{l}\text { Whole } \\
\text { population }\end{array}$ & $\begin{array}{l}\text { Women who trial labor } \\
\text { after caesarean } \\
\text { section }\end{array}$ & Medical records & 1119 & 686 & $63.0 \%$ \\
\hline Sole KB. Et al. (75) & 01/1999 - 12/2014 & Norway, National & $\begin{array}{l}\text { Whole } \\
\text { population }\end{array}$ & $\begin{array}{l}\text { Women with singleton } \\
\text { pregnancies }\end{array}$ & Medical records & 907048 & 14200 & $1.57 \%$ \\
\hline Magnus MA. et al. (76) & 01/2009 - 12/2013 & Norway, National & $\begin{array}{l}\text { Whole } \\
\text { population }\end{array}$ & General population & Medical records & 162343 & 5938 & $3.7 \%$ \\
\hline \multicolumn{9}{|l|}{ Sweden } \\
\hline Lindqvist M. et al. (77) & $2011-2012$ & Sweden, National & $\begin{array}{l}\text { Whole } \\
\text { population }\end{array}$ & General population & Medical records & 181292 & 2548 & $1.4 \%$ \\
\hline Nilsson C. et al. (78) & $2012-2013$ & Sweden, National & $\begin{array}{l}\text { Whole } \\
\text { population }\end{array}$ & General population & WHO 1999 & 7491 & 210 & $2.8 \%$ \\
\hline Stokkeland K. et al. (79) & $2006-2011$ & Sweden, National & $\begin{array}{l}\text { Whole } \\
\text { population }\end{array}$ & General population & Medical records & 576642 & 6343 & $1.0 \%$ \\
\hline Sundelin HEK. et al. (80) & $2006-2014$ & Sweden, National & $\begin{array}{l}\text { Whole } \\
\text { population }\end{array}$ & General population & Medical records & 877742 & 9919 & $1.1 \%$ \\
\hline Stogianni A. et al. (81) & $2009-2012$ & Sweden, Kronoberg & $\begin{array}{l}\text { Whole } \\
\text { population }\end{array}$ & General population & Medical records & 280 & 97 & $34.6 \%$ \\
\hline Crump C. et al. (82) & $1973-2014$ & Sweden, National & $\begin{array}{l}\text { Whole } \\
\text { population }\end{array}$ & General population & Medical records & 4186615 & 34255 & $0.8 \%$ \\
\hline Hilden K. et al. (83) & $1998-2012$ & Sweden, National & $\begin{array}{l}\text { Whole } \\
\text { population }\end{array}$ & General population & Medical records & 1294006 & 14833 & $1.0 \%$ \\
\hline
\end{tabular}


TABLE 2 | Continued

\begin{tabular}{|c|c|c|c|c|c|c|c|c|}
\hline Author (Ref) & $\begin{array}{l}\text { Duration of data } \\
\text { collection }\end{array}$ & City & $\begin{array}{l}\text { Sampling } \\
\text { strategy }\end{array}$ & Population & $\begin{array}{l}\text { Ascertainment } \\
\text { method }\end{array}$ & $\begin{array}{l}\text { Tested } \\
\text { sample }\end{array}$ & $\begin{array}{c}\text { GDM } \\
\text { Positive }\end{array}$ & $\begin{array}{c}\text { Prev. } \\
(\%)\end{array}$ \\
\hline Khashan AS. et al. (84) & $1982-2012$ & Sweden, National & $\begin{array}{l}\text { Whole } \\
\text { population }\end{array}$ & General population & Medical records & 1292792 & 4967 & $0.4 \%$ \\
\hline Liu C. et al. (85) & $2014-2017$ & Sweden, National & $\begin{array}{l}\text { Whole } \\
\text { population }\end{array}$ & Refugees & Medical records & 31897 & 1148 & $3.6 \%$ \\
\hline \multicolumn{9}{|l|}{ United Kingdom } \\
\hline Farrar D. et al. (86) & $2008-2010$ & UK, Bradford & $\begin{array}{l}\text { Whole } \\
\text { population }\end{array}$ & General population & WHO 1999 & 11516 & 1132 & $10 \%$ \\
\hline \multirow[t]{2}{*}{ West J et al. (87) } & 03/2007 - 12/2010 & UK, Bradford & Consecutive & $\begin{array}{l}\text { Caucasian British/lrish } \\
\text { women }\end{array}$ & WHO 1999 & 3503 & 172 & $4.9 \%$ \\
\hline & & & & Pakistani women & & 2656 & 406 & $15.3 \%$ \\
\hline Syngelaki A. et al. (88) & 03/2006 - 07/2013 & $\begin{array}{l}\text { UK, London and } \\
\text { Gillingham }\end{array}$ & Unclear & General population & Mixed methods & 75161 & 1827 & $2.4 \%$ \\
\hline Poston L. et al. (89) & $03 / 2009-06 / 2014$ & $\begin{array}{l}\text { UK, London, } \\
\text { Bradford, Glasgow, } \\
\text { Manchester, } \\
\text { Newcastle, } \\
\text { Sunderland }\end{array}$ & $\begin{array}{l}\text { Random } \\
\text { sampling }\end{array}$ & Obese women & IADPSG & 1280 & 332 & $26 \%$ \\
\hline Sovio U. et al. (90) & 08/2008 - 07/2012 & UK, Cambridge & Unclear & Nulliparous women & Mixed methods & 4069 & 171 & $4.2 \%$ \\
\hline \multirow[t]{2}{*}{ Murphy NM. et al. (91) } & 05/2007-02/2011 & $\begin{array}{l}\text { UK, London, } \\
\text { Manchester, Cork, }\end{array}$ & Unclear & $\begin{array}{l}\text { Women at high risk of } \\
\text { GDM }\end{array}$ & Mixed methods & 395 & 35 & $8.9 \%$ \\
\hline & & Leeds & & $\begin{array}{l}\text { Pregnant women } \\
\text { without risk of GDM }\end{array}$ & & 261 & 20 & $7.7 \%$ \\
\hline White SL. et al. (92) & $2009-2014$ & UK & Unclear & Obese women & IADPSG & 1303 & 337 & $25.9 \%$ \\
\hline Hanna FW. et al. (93) & 02/2010 - 12/2013 & UK & Unclear & General population & NICE 2015 & 6930 & 967 & $13.7 \%$ \\
\hline Panaitescu AM. et al. (94) & 03/2006 - 11/2015 & UK, London & Unclear & General population & WHO 1999 & 107788 & 2542 & $2.4 \%$ \\
\hline Hall E. et al., (95) & $05 / 2017-08 / 2017$ & UK, London & $\begin{array}{l}\text { Whole } \\
\text { population }\end{array}$ & General population & NICE 2015 & 1267 & 264 & $21 \%$ \\
\hline Balani J. et al. (96) & $2010-2011$ & UK, Surrey & Unclear & Obese women & WHO 1999 & 302 & 72 & $23.8 \%$ \\
\hline Nzelu D. et al. (97) & $2011-2016$ & UK, London & Consecutive & $\begin{array}{l}\text { Pregnant women with } \\
\text { pregnancy induced } \\
\text { hypertension }\end{array}$ & NICE 2015 & 773 & 93 & $12 \%$ \\
\hline Vieira MC. et al. (98) & 03/2009-06/2014 & UK, London & $\begin{array}{l}\text { Whole } \\
\text { population }\end{array}$ & Obese women & IADPSG & 824 & 241 & $29.6 \%$ \\
\hline Wagnild JM. et al. (99) & $02 / 2017-08 / 2017$ & $\begin{array}{l}\text { UK, Northeast } \\
\text { England }\end{array}$ & Consecutive & $\begin{array}{l}\text { Women at high risk of } \\
\text { GDM }\end{array}$ & NICE 2015 & 326 & 31 & $16.5 \%$ \\
\hline
\end{tabular}

ADA, American Diabetes Association; BMI, body mass-index; DM, diabetes mellitus; GDM, gestational diabetes mellitus; IADPSG, International Association of the Diabetes and Pregnancy Study Groups; NICE, National Institute for Health and Care Excellence; UK, United Kingdom; WHO, World Health Organization.

Sweden (48\%), France (20.0\%), and Norway (8.6\%). From the represented countries in our analysis, Sweden (Northern Europe) shows the lowest weighted GDM prevalence of $1.8 \%$ (95\% CI: 1.5-2.2, $I^{2}, 99.9 \%$ ) (Table 5). The highest observed nationalbased prevalence of $66.1 \%$ from a single study in the Republic of Moldova has contributed to the observed highest weighted GDM prevalence in the Eastern Europe sub-region (Table 5).

\section{Sub-Regional Weighted GDM Prevalence}

The highest sub-regional weighted GDM prevalence observed in the three Eastern European countries (31.5\%, 95\% CI: 19.8-44.6, $\left.I^{2}, 98.9 \%\right)$, followed by $12.3 \%$ (95\% CI:10.9-13.9, $\left.I^{2}, 99.6 \%\right)$ in Southern Europe, 10.7\% (95\% CI: 9.5-12.0, $I^{2}$, 99.9\%) in Western Europe, and $8.9 \%$ (95\% CI: $\left.7.9-10.0, I^{2}, 100.0 \%\right)$ in Northern Europe.

\section{Sub-Group Analysis}

The weighted prevalence of GDM was significantly higher in pregnant women $\geq 30$ years old $\left(15.4 \%, \mathrm{I}^{2}, 99.8 \%\right)$ compared with
$15-29$ years old women $\left(7.2 \%, I^{2}, 99.6 \%\right)$, in their third $(18.4 \%$, $\left.\mathrm{I}^{2}, 99.8 \%\right)$ compared with second trimester $\left(12.5 \%, I^{2}, 99.9 \%\right)$ of pregnancy, in obese $\left(23.1 \%, I^{2}, 98.3 \%\right)$ and overweight $(7.8 \%, \mathrm{I} 2$, $99.5 \%)$ compared with normal weight $\left(3.4 \%, I^{2}, 99.4 \%\right)$ pregnant women.

This observation was comparable in the four sub-regions, whenever data was available. In the Northern European subregion that comprised $48.0 \%$ of the GDM prevalence studies and tested $66.0 \%$ of the pregnant women in Europe, the weighted prevalence of GDM was 1.86-time higher in pregnant women $\geq 30$ years old $\left(13.4 \%, \mathrm{I}^{2}, 99.7 \%\right)$ compared with younger women $\left(7.2 \%, I^{2}, 99.7 \%\right), 1.83$-time higher in the third trimester $(18.0 \%$, 95\% CI: $\left.10.0-27.7, I^{2}, 99.8 \%\right)$ compared with the second trimester (9.8\%, 95\% CI: 7.6-12.2, $\left.I^{2}, 99.9 \%\right)$, 4.2-time and 14.1-time higher in obese $\left(31.1 \%, 95 \%\right.$ CI: $\left.26.5-35.8, I^{2}, 0.0 \%\right)$ compared with overweight (7.4\%) and normal weight $(2.2 \%)$ women, respectively. In all sub-regions, there was a significant variation $(\mathrm{p}<0.001)$ in the weighted GDM prevalence between the used GDM ascertainment guidelines (Supplementary Table S3). 
TABLE 3 | Baseline studies characteristics from Western Europe.

\begin{tabular}{|c|c|c|c|c|c|c|c|c|}
\hline Author (Ref) & $\begin{array}{l}\text { Duration of data } \\
\text { collection }\end{array}$ & City & $\begin{array}{l}\text { Sampling } \\
\text { strategy }\end{array}$ & Population & $\begin{array}{l}\text { Ascertainment } \\
\text { method }\end{array}$ & $\begin{array}{l}\text { Tested } \\
\text { sample }\end{array}$ & $\begin{array}{c}\text { GDM } \\
\text { Positive }\end{array}$ & $\begin{array}{l}\text { Prev. } \\
(\%)\end{array}$ \\
\hline \multicolumn{9}{|l|}{ Austria } \\
\hline $\begin{array}{l}\text { Bozkurt L. et al. } \\
(100)\end{array}$ & $2010-2014$ & Austria, Vienna & Unclear & $\begin{array}{l}\text { General population with OGTT at } \\
16 \text { weeks }\end{array}$ & IADPSG & 221 & 81 & $38.3 \%$ \\
\hline $\begin{array}{l}\text { Tramontana A. } \\
\text { et al. (101) }\end{array}$ & 01/2010- 11/2013 & Austria, Vienna & $\begin{array}{l}\text { Whole } \\
\text { population }\end{array}$ & General population & IADPSG & 4948 & 209 & $4.2 \%$ \\
\hline $\begin{array}{l}\text { Tramontana A. } \\
\text { et al. (102) }\end{array}$ & & Austria, Vienna & $\begin{array}{l}\text { Whole } \\
\text { population }\end{array}$ & Women with high-risk pregnancies & IADPSG & 382 & 170 & $44.5 \%$ \\
\hline $\begin{array}{l}\text { Koninger A. et al. } \\
(103)\end{array}$ & $2009-2018$ & Austria, Essen & $\begin{array}{l}\text { Whole } \\
\text { population }\end{array}$ & $\begin{array}{l}\text { Women with polycystic ovary } \\
\text { syndrome }\end{array}$ & GDDD & 63 & 29 & $46 \%$ \\
\hline $\begin{array}{l}\text { Weiss C. et al. } \\
(104)\end{array}$ & $01 / 2013-12 / 2015$ & Austria, Linz & $\begin{array}{l}\text { Whole } \\
\text { population }\end{array}$ & Singleton pregnancies & WHO 2013 & 3293 & 553 & $16.8 \%$ \\
\hline \multicolumn{9}{|l|}{ Belgium } \\
\hline $\begin{array}{l}\text { Benhalima K. et al. } \\
(105)\end{array}$ & $01 / 2010-12 / 2013$ & $\begin{array}{l}\text { Belgium, Leuven, } \\
\text { Aalst }\end{array}$ & $\begin{array}{l}\text { Whole } \\
\text { population }\end{array}$ & General population & $\begin{array}{l}\text { Carpenter- } \\
\text { Coustan }\end{array}$ & 14661 & 601 & $4.1 \%$ \\
\hline $\begin{array}{l}\text { De Munck N. et al. } \\
\text { (106) }\end{array}$ & 03/2010 - 08/2014 & Belgium, Brussels & $\begin{array}{l}\text { Whole } \\
\text { population }\end{array}$ & $\begin{array}{l}\text { Ocyte recipient with use of closed } \\
\text { vitrification }\end{array}$ & Mix method & 112 & 13 & $11.6 \%$ \\
\hline \multicolumn{9}{|l|}{ France } \\
\hline $\begin{array}{l}\text { Grunewald D. } \\
\text { et al. (107) }\end{array}$ & $2008-2013$ & France, Paris & Unclear & $\begin{array}{l}\text { Pregnant women with cystic } \\
\text { fibrosis }\end{array}$ & Medical records & 23 & 2 & $8.7 \%$ \\
\hline $\begin{array}{l}\text { Miailhe G. et al. } \\
(108)\end{array}$ & 04/2011 - 02/2012 & France, Paris & $\begin{array}{l}\text { Whole } \\
\text { population }\end{array}$ & Singleton pregnancies & IADPSG & 2187 & 309 & $14 \%$ \\
\hline $\begin{array}{l}\text { Goueslard K. et al. } \\
\text { (109) }\end{array}$ & $2007-2013$ & France, National & $\begin{array}{l}\text { Whole } \\
\text { population }\end{array}$ & General population & Medical records & 1515387 & 62958 & $4.14 \%$ \\
\hline $\begin{array}{l}\text { Regnault N. et al. } \\
(110)\end{array}$ & 2013 & France, Bondy & $\begin{array}{l}\text { Whole } \\
\text { population }\end{array}$ & General population & Medical records & 788494 & 67810 & $8.6 \%$ \\
\hline $\begin{array}{l}\text { Mortier I. et al. } \\
(111)\end{array}$ & 01/2011-07/2012 & France, Marseille & $\begin{array}{l}\text { Whole } \\
\text { population }\end{array}$ & Singleton pregnancies & IADPSG & 444 & 60 & $13.5 \%$ \\
\hline $\begin{array}{l}\text { Boudet-Berquier J. } \\
\text { et al. (112) }\end{array}$ & 01/2012 - 04/2014 & France, National & $\begin{array}{l}\text { Random } \\
\text { sampling }\end{array}$ & General population & Mixed methods & 3204 & 247 & $7.7 \%$ \\
\hline $\begin{array}{l}\text { Billionnet C. et al. } \\
\text { (113) }\end{array}$ & 2012 & France, National & $\begin{array}{l}\text { Whole } \\
\text { population }\end{array}$ & General population & Medical records & 796346 & 57629 & $7.24 \%$ \\
\hline \multirow[t]{2}{*}{$\begin{array}{l}\text { Mitanchez D. et al. } \\
(114)\end{array}$} & 08/2010 - 03/2013 & France, Paris & Unclear & $\begin{array}{l}\text { Singleton pregnancy in obese } \\
\text { women }\end{array}$ & IADPSG & 226 & 99 & $43.8 \%$ \\
\hline & & & & $\begin{array}{l}\text { Singleton pregnancy in normal } \\
\text { weight women }\end{array}$ & & 222 & 41 & $18.4 \%$ \\
\hline Marie C. et al. & 2006 & Auvergne, France & Whole & General population & Carpenter- & 1175 & 73 & $6.2 \%$ \\
\hline$(115)$ & 2010 & & population & & Coustan & 2840 & 156 & $5.5 \%$ \\
\hline $\begin{array}{l}\text { Preaubert L. et al. } \\
\text { (116) }\end{array}$ & 01/2010 - 12/2016 & France, Paris & $\begin{array}{l}\text { Whole } \\
\text { population }\end{array}$ & $\begin{array}{l}\text { Ocyte recipient with use of closed } \\
\text { vitrification }\end{array}$ & IADPSG & 247 & 39 & $15.8 \%$ \\
\hline $\begin{array}{l}\text { Soomro MH. et al. } \\
(117)\end{array}$ & 03/2003 - 01/2006 & $\begin{array}{l}\text { France, Poitiers } \\
\text { and Nancy }\end{array}$ & $\begin{array}{l}\text { Whole } \\
\text { population }\end{array}$ & $\begin{array}{l}\text { Women with blood-biomarkers to } \\
\text { study heavy metals }\end{array}$ & $\begin{array}{l}\text { Carpenter- } \\
\text { Coustan }\end{array}$ & 623 & 4 & $7.1 \%$ \\
\hline \multicolumn{9}{|l|}{ Germany } \\
\hline $\begin{array}{l}\text { Stuber TN. et al. } \\
(118)\end{array}$ & $2006-2011$ & $\begin{array}{l}\text { Germany, } \\
\text { Wurzburg }\end{array}$ & $\begin{array}{l}\text { Whole } \\
\text { population }\end{array}$ & General population & Medical records & 2810 & 264 & $9.4 \%$ \\
\hline $\begin{array}{l}\text { Beyerlein A. et al. } \\
(119)\end{array}$ & $2008-2014$ & Germany, Bavaria & Consecutive & General population & Medical records & 173718 & 6427 & $3.7 \%$ \\
\hline \multirow{2}{*}{$\begin{array}{l}\text { Tamayo T. et al. } \\
(120)\end{array}$} & 07/2012 - 06/2013 & Germany, North & Consecutive & General population & IADPSG & 153302 & 9229 & $6.0 \%$ \\
\hline & 07/2013 - 06/2014 & Rhine & & & & 158839 & 10817 & $6.8 \%$ \\
\hline $\begin{array}{l}\text { Melchior H. et al. } \\
(121)\end{array}$ & $01 / 2014-12 / 2015$ & Germany, National & $\begin{array}{l}\text { Whole } \\
\text { population }\end{array}$ & General population & Medical records & 567191 & 74869 & $13.2 \%$ \\
\hline $\begin{array}{l}\text { Köninger A. et al. } \\
(122)\end{array}$ & $2014-2016$ & Germany, Essen & Unclear & Singleton pregnancies & $\begin{array}{l}\text { German Diabetes } \\
\text { Association }\end{array}$ & 105 & 29 & 27.64 \\
\hline $\begin{array}{l}\text { Pahlitzsch TMJ. } \\
\text { et al. (123) } \\
\text { Netherlands }\end{array}$ & $2001-2017$ & Germany, Solingen & $\begin{array}{l}\text { Whole } \\
\text { population }\end{array}$ & $\begin{array}{l}\text { Mothers of macrocosmic } \\
\text { newborns }\end{array}$ & Medical records & 2277 & 87 & $3.8 \%$ \\
\hline $\begin{array}{l}\text { Lamain-de-Ruiter } \\
\text { ML (124). }\end{array}$ & $12 / 2010-01 / 2014$ & Netherlands & Unclear & General population & Mixed method & 3723 & 181 & $4.9 \%$ \\
\hline $\begin{array}{l}\text { Koning SH. et al. } \\
(125)\end{array}$ & 01/2011 - 09/2016 & $\begin{array}{l}\text { Netherlands, } \\
\text { Groningen }\end{array}$ & $\begin{array}{l}\text { Whole } \\
\text { population }\end{array}$ & $\begin{array}{l}\text { Pregnant women with at least one } \\
\text { risk factors for GDM }\end{array}$ & WHO 2013 & 10642 & 3364 & $31.6 \%$ \\
\hline \multirow[t]{2}{*}{$\begin{array}{l}\text { De Wilde MA. et al. } \\
\text { (126) }\end{array}$} & 04/2008 - 04/2012 & Netherlands & Unclear & $\begin{array}{l}\text { Women with polycystic ovarian } \\
\text { syndrome }\end{array}$ & ADA 2004 & 188 & 43 & $23 . \%$ \\
\hline & $12 / 2012-12 / 2013$ & & & Singleton pregnancies & WHO 1999 & 2889 & 129 & $4.5 \%$ \\
\hline
\end{tabular}


TABLE 3 | Continued

\begin{tabular}{|c|c|c|c|c|c|c|c|c|}
\hline Author (Ref) & $\begin{array}{l}\text { Duration of data } \\
\text { collection }\end{array}$ & City & $\begin{array}{l}\text { Sampling } \\
\text { strategy }\end{array}$ & Population & $\begin{array}{l}\text { Ascertainment } \\
\text { method }\end{array}$ & $\begin{array}{l}\text { Tested } \\
\text { sample }\end{array}$ & $\begin{array}{c}\text { GDM } \\
\text { Positive }\end{array}$ & $\begin{array}{c}\text { Prev. } \\
(\%)\end{array}$ \\
\hline \multicolumn{9}{|l|}{ Switzerland } \\
\hline $\begin{array}{l}\text { Mosimann B. et al. } \\
(127)\end{array}$ & $01 / 2014-12 / 2014$ & Switzerland, Bern & Consecutive & General population & Mixed method & 328 & 51 & $15.5 \%$ \\
\hline $\begin{array}{l}\text { Amylidi S. et al. } \\
\text { (128) }\end{array}$ & 06/2011 - 11/2012 & Switzerland, Bern & $\begin{array}{l}\text { Whole } \\
\text { population }\end{array}$ & $\begin{array}{l}\text { Pregnant women with at least one } \\
\text { risk factors for GDM }\end{array}$ & ADA 2016 & 218 & 32 & $14.7 \%$ \\
\hline $\begin{array}{l}\text { Ryser Rüetschi J. } \\
\text { et al. (129) }\end{array}$ & $10 / 2010-04 / 2012$ & $\begin{array}{l}\text { Switzerland, } \\
\text { Geneva and Basel }\end{array}$ & Consecutive & General population & IADSPG & 2298 & 251 & $10.9 \%$ \\
\hline $\begin{array}{l}\text { Horsch A. et al. } \\
(130)\end{array}$ & $11 / 2012$ * 07/2013 & $\begin{array}{l}\text { Switzerland, } \\
\text { Lausanne }\end{array}$ & $\begin{array}{l}\text { Whole } \\
\text { population }\end{array}$ & General population & Mixed method & 203 & 39 & $19.2 \%$ \\
\hline $\begin{array}{l}\text { Savopol H. et al. } \\
\text { (131) }\end{array}$ & $01 / 2014-12 / 2015$ & & $\begin{array}{l}\text { Whole } \\
\text { population }\end{array}$ & General population & IADSPG & 502 & 159 & $31.7 \%$ \\
\hline
\end{tabular}

ADA, American Diabetes Association; DM, diabetes mellitus; GDDD, Deutsche Gesellschaft fur gynakologie und Geburtshilfe; HBV, Hepatitis B virus, HIV, Human Immunodeficiency virus; IADPSG, International Association of the Diabetes and Pregnancy Study Groups; OGTT, oral glucose tolerance test; WHO, World Health Organization.

\section{Risk of Bias (RoB)}

The results of the four RoB domains assessed and the six quality of evidence items from NIH are presented in (Figure 2). Overall, the RoB and quality of evidence showed a significant low RoB with domains like the study population and research question having $100 \%$ of high quality of evidence. Recruitment and outcomes measurement were also rated with high quality of evidence in $97 \%$, while sample size justification was unclear for $70 \%$ of the studies. Regarding RoB, GDM ascertainment and precision were low for $4 \%$ and $5 \%$, respectively. While the response rate and sampling methodology were considered high for $14 \%$ and $10 \%$, respectively (Figure 2).

\section{Publication Bias}

Graphically, the funnel plot shows a potential of publication bias and small-study effect (Egger's test, $p<0.001$ ) on the estimated pooled prevalence (Supplementary Figure S1).

\section{DISCUSSION}

\section{Summary of Evidence}

This systematic review and meta-analysis research summarizes the prevalence of GDM in Europe based on 133 reports comprising data of 254 single studies reported between 2014 and 2019 in 24 countries. Most of these studies were from Italy and the United Kingdom. The overall estimated prevalence of GDM in the 24 countries from the entire European Region was lower (10.9\%, 95\% CI: 10.0-11.8, $\left.I^{2}: 100 \%\right)$ than the estimates reported by the International Diabetes Federation (IDF) for 2019 $(16.3 \%)(168)$ and higher than a previous meta-analysis $(5.4 \%$, 95\% CI: 3.8-7.8) conducted by Eades and colleagues (22). Differences in the population estimates (and countries) might explain the variation between the reports. IDF has included data of 39 countries and only for women aged 20-45 years old (168) and Eades and colleagues included only 12 countries (22). A descriptive study revising the global GDM prevalence points to Europe as the region with the lowest GDM prevalence with a median of 6.1 (range $1.8 \%-31.0 \%$ ) (169), in our study, the median estimate was 9.9 (range $0.2 \%-78 \%$ ).

Considering the four sub-regions of Europe, the Eastern region presented the highest GDM prevalence (31.5\%, 95\% CI: 19.8-44.6, $\left.I^{2}: 98.9 \%\right)$, followed by Southern Europe (12.3\%, 95\% CI: $\left.10.9-13.9, I^{2}: 99.6 \%\right)$, Western Europe (10.7\%, 95\% CI: $9.5-$ 12.0, $I^{2}: 99.9 \%$ ), and Northern Europe (8.9\%, 95\% CI: 7.9-10.0, $\left.I^{2}: 100\right)$. A review of the literature from 2000-2009 is consistent with these results presenting the lowest GDM prevalence for the European northern or Atlantic seaboard countries in comparison with the Southern or Mediterranean countries (170). The Eastern (and Southern regions were also the two regions with the smallest number of studies included, $4.5 \%$ and $27.1 \%$ respectively, due to the lack of identified reports from these countries. These results highlight the need for good quality and standardized epidemiological studies in these two regions, not to mention the 25 countries that are not represented in our study. We have assessed full-text studies from some countries like Albania and Portugal that were potentially eligible to be considered but as the GDM ascertainment criteria was not clear, therefore they were excluded for not meeting our criteria.

The Republic of Moldova has the highest GDM prevalence across the entire region (66.1\%, 95\% CI 19.8-44.6\%, I²: 98.9\%), followed by Poland, Austria, Cyprus, and Malta. Sweden has the lowest GDM prevalence followed by Belgium, Norway, Croatia, and Denmark. The IDF 2019 Diabetes Atlas presents GDM prevalence for 12 countries in the region and their estimated prevalence is within our confidence interval for France, Ireland, Netherlands, Poland, and Sweden (168). For Norway, Spain, and the UK their estimates are higher than ours. These findings may suggest the recent higher reported rates for GDM prevalence compared with previous years as our review comprises data from 2014-2019 and there is just for 2019.

In women with a history of GDM, lifestyle interventions and medical treatment decreased the progression of T2DM by up to $40 \%$ (171). Therefore, GDM becomes a public health priority issue as it poses a significant health burden, not only to these pregnancies but also to the future health of both mothers and offspring. In this way, the diagnosis and management of GDM can represent an opportunity for intervention to reduce the 
TABLE 4 | Baseline studies characteristics from Southern Europe.

\begin{tabular}{|c|c|c|c|c|c|c|c|c|}
\hline $\begin{array}{l}\text { Author } \\
\text { (Ref) }\end{array}$ & $\begin{array}{l}\text { Duration of data } \\
\text { collection }\end{array}$ & City & $\begin{array}{l}\text { Sampling } \\
\text { strategy }\end{array}$ & Population & $\begin{array}{l}\text { Ascertainment } \\
\text { method }\end{array}$ & $\begin{array}{l}\text { Tested } \\
\text { sample }\end{array}$ & $\begin{array}{c}\text { GDM } \\
\text { Positive }\end{array}$ & $\begin{array}{c}\text { Prev. } \\
\text { (\%) }\end{array}$ \\
\hline \multicolumn{9}{|l|}{ Croatia } \\
\hline $\begin{array}{l}\text { Djakovicí l. } \\
\text { et al. (132) }\end{array}$ & $2011-2012$ & $\begin{array}{l}\text { Croatia, } \\
\text { Zagreb }\end{array}$ & Consecutive & General population & $\begin{array}{l}\text { HAPO study } \\
\text { guidelines }\end{array}$ & 6407 & 593 & $9.3 \%$ \\
\hline $\begin{array}{l}\text { Djelmis J. } \\
\text { et al. (133) }\end{array}$ & $2012-2014$ & Croatia & Unclear & Singleton pregnancies & $\begin{array}{l}\text { IADSPG } \\
\text { NICE } 2015\end{array}$ & $\begin{array}{l}4646 \\
4646\end{array}$ & $\begin{array}{l}1074 \\
826\end{array}$ & $\begin{array}{l}23.1 \% \\
17.8 \%\end{array}$ \\
\hline $\begin{array}{l}\text { Erjavec K. } \\
\text { et al. (134) }\end{array}$ & $\begin{array}{l}2010 \\
2014\end{array}$ & $\begin{array}{l}\text { Croatia, } \\
\text { National }\end{array}$ & Consecutive & General population & $\begin{array}{l}\text { WHO } 1999 \\
\text { IADSPG }\end{array}$ & $\begin{array}{l}42656 \\
39092\end{array}$ & $\begin{array}{c}953 \\
1829\end{array}$ & $\begin{array}{l}2.2 \% \\
4.6 \%\end{array}$ \\
\hline $\begin{array}{l}\text { Vince K, } \\
\text { et al. (135) } \\
\text { Cyprus }\end{array}$ & 2011 & $\begin{array}{l}\text { Croatia, } \\
\text { National }\end{array}$ & Consecutive & General population & IADSPG & 40641 & 1181 & $2.9 \%$ \\
\hline $\begin{array}{l}\text { Inancli SS } \\
\text { et al. (136) } \\
\text { Greece }\end{array}$ & $11 / 2013-04 / 2014$ & $\begin{array}{l}\text { Cyprus, } \\
\text { National }\end{array}$ & Consecutive & Turkish Cypriot & $\begin{array}{l}\text { National Diabetes } \\
\text { Data Group }\end{array}$ & 230 & 45 & $19.6 \%$ \\
\hline $\begin{array}{l}\text { Vassilaki M. } \\
\text { et al. (137) } \\
\text { Italy }\end{array}$ & 02/2007 - 02/2008 & $\begin{array}{l}\text { Greece, } \\
\text { Crete }\end{array}$ & Convenience & General population & Carpenter-Coustan & 1122 & 102 & $9.1 \%$ \\
\hline $\begin{array}{l}\text { Trotta F. } \\
\text { et al. (138) }\end{array}$ & 10/2009 - 09/2010 & $\begin{array}{l}\text { Italy, } \\
\text { Lombardy }\end{array}$ & $\begin{array}{l}\text { Whole } \\
\text { population }\end{array}$ & General population & Medical records & 86171 & 1921 & $2.3 \%$ \\
\hline $\begin{array}{l}\text { Pintaudi B. } \\
\text { et al. (139) }\end{array}$ & $05 / 2010-10 / 2011$ & $\begin{array}{l}\text { Italy, } \\
\text { Messina }\end{array}$ & Consecutive & Caucasian women & IADSPG & 1015 & 113 & $11.1 \%$ \\
\hline $\begin{array}{l}\text { Caserta D. } \\
\text { et al. (140) }\end{array}$ & $01 / 2007-06 / 2011$ & Italy, Rome & $\begin{array}{l}\text { Whole } \\
\text { population }\end{array}$ & $\begin{array}{l}\text { Twin pregnancies } \\
\text { Twin pregnancies with assisted conception }\end{array}$ & Medical records & $\begin{array}{l}207 \\
138\end{array}$ & $\begin{array}{c}6 \\
14\end{array}$ & $\begin{array}{c}2.9 \% \\
10.1 \%\end{array}$ \\
\hline $\begin{array}{l}\text { Lacaria E. } \\
\text { et al. (141) }\end{array}$ & $01 / 2012-13 / 2013$ & $\begin{array}{l}\text { Italy, Pisa } \\
\text { and Livorno }\end{array}$ & Consecutive & Caucasian women & IADSPG & 2497 & 279 & $11.1 \%$ \\
\hline $\begin{array}{l}\text { D'Anna R. } \\
\text { et al. (142) }\end{array}$ & $01 / 2011-04 / 2014$ & $\begin{array}{l}\text { Italy, } \\
\text { Messina and } \\
\text { Modina }\end{array}$ & $\begin{array}{l}\text { Random } \\
\text { sampling }\end{array}$ & Obese women & IADSPG & 241 & 51 & $23.8 \%$ \\
\hline $\begin{array}{l}\text { Pinzauti S. } \\
\text { et al. (143) }\end{array}$ & $01 / 2010-12 / 2014$ & $\begin{array}{l}\text { Italy, } \\
\text { Florence, } \\
\text { and Siena }\end{array}$ & $\begin{array}{l}\text { Whole } \\
\text { population }\end{array}$ & Twin pregnancies with assisted conception & Mixed method & 430 & 30 & $6.9 \%$ \\
\hline $\begin{array}{l}\text { Capula C. } \\
\text { et al. (144) }\end{array}$ & $08 / 2011-01 / 2015$ & $\begin{array}{l}\text { Italy, } \\
\text { Catanzaro }\end{array}$ & Convenience & Healthy pre-pregnancy women & IADSPG & 3974 & 1066 & $26.8 \%$ \\
\hline Santamaria & $01 / 2012-12 / 2014$ & Italy, & Convenience & Overweight Caucasian & ADA 2011 & 102 & 28 & $27.5 \%$ \\
\hline $\begin{array}{l}\text { A. et al. } \\
(145)\end{array}$ & & $\begin{array}{l}\text { Messina and } \\
\text { Modena }\end{array}$ & & Overweight Caucasian receiving Myo-inositol & & 95 & 11 & $11.6 \%$ \\
\hline $\begin{array}{l}\text { Bianchi C. } \\
\text { et al. (146) }\end{array}$ & $01 / 2010-03 / 2015$ & Italy, Pisa & Unclear & General population & Medical records & 1198 & 476 & $39.7 \%$ \\
\hline $\begin{array}{l}\text { Di Cianni G. } \\
\text { et al. (147) }\end{array}$ & $01 / 2015-12 / 2015$ & $\begin{array}{l}\text { Italy, } \\
\text { Tuscany }\end{array}$ & $\begin{array}{l}\text { Whole } \\
\text { population }\end{array}$ & General population & Medical records & 17606 & 2000 & $11.4 \%$ \\
\hline Bordi et al. & $01 / 2001-06 / 2015$ & Italy, Rome & Whole & Twin pregnancies with assisted conception & Medical records & 450 & 38 & $8.4 \%$ \\
\hline$(148)$ & & & population & Twin pregnancies & & 647 & 18 & $2.8 \%$ \\
\hline $\begin{array}{l}\text { Chiefari E. } \\
\text { et al. (149) }\end{array}$ & $08 / 2011-12 / 2016$ & $\begin{array}{l}\text { Italy, } \\
\text { Cantazaro }\end{array}$ & Unclear & General population & $\begin{array}{l}\text { Italian Minister } \\
\text { Guidelines }\end{array}$ & 5473 & 1559 & $28.5 \%$ \\
\hline $\begin{array}{l}\text { Cozzolino } \\
\text { M, et al. } \\
(150)\end{array}$ & $01 / 2010-01 / 2016$ & $\begin{array}{l}\text { Italy, } \\
\text { Florence }\end{array}$ & $\begin{array}{l}\text { Whole } \\
\text { population }\end{array}$ & Multiple pregnancies & IADSPG & 656 & 99 & $15.1 \%$ \\
\hline \multirow[t]{2}{*}{$\begin{array}{l}\text { Bruno R. } \\
\text { et al. (151) }\end{array}$} & \multirow[t]{2}{*}{ 02/2013 - 06/2014 } & \multirow[t]{2}{*}{$\begin{array}{l}\text { Italy, } \\
\text { Modena }\end{array}$} & \multirow[t]{2}{*}{ Unclear } & $\begin{array}{l}\text { Singleton pregnancies of overweight/obese } \\
\text { women with prescribed personalized dietary } \\
\text { intervention }\end{array}$ & IADSPG & 62 & 23 & $37.1 \%$ \\
\hline & & & & Singleton pregnancies of obese women & & 69 & 13 & $18.8 \%$ \\
\hline $\begin{array}{l}\text { Bianchi C. } \\
\text { et al. (152) }\end{array}$ & $01 / 2013-12 / 2015$ & Italy, Pisa & $\begin{array}{l}\text { Whole } \\
\text { population }\end{array}$ & General population & $\begin{array}{l}\text { Italian National } \\
\text { Guidelines }\end{array}$ & 1338 & 534 & 39.95 \\
\hline $\begin{array}{l}\text { Meregaglia } \\
\text { M. et al. } \\
(153)\end{array}$ & $01 / 2014-12 / 2014$ & $\begin{array}{l}\text { Italy, } \\
\text { National }\end{array}$ & $\begin{array}{l}\text { Whole } \\
\text { population }\end{array}$ & General population & Medical records & 44402 & 11540 & $10.9 \%$ \\
\hline $\begin{array}{l}\text { Quaresima } \\
\text { P. et al. } \\
(154)\end{array}$ & $01 / 2015-12 / 2016$ & $\begin{array}{l}\text { Italy, } \\
\text { Catanzaro }\end{array}$ & Consecutive & General population & IADSPG & 1413 & 451 & $31.8 \%$ \\
\hline \multirow[t]{2}{*}{$\begin{array}{l}\text { Gerli S. } \\
\text { et al. (155) }\end{array}$} & \multirow[t]{2}{*}{$01 / 2011-12 / 2013$} & \multirow[t]{2}{*}{$\begin{array}{l}\text { Italy, } \\
\text { National }\end{array}$} & \multirow[t]{2}{*}{$\begin{array}{l}\text { Whole } \\
\text { population }\end{array}$} & $\begin{array}{l}\text { Women in Robson class } 1 \text { according to the Ten } \\
\text { Group Classification System }\end{array}$ & IADSPG & 7693 & 132 & $1.7 \%$ \\
\hline & & & & $\begin{array}{l}\text { Women in Robson class } 3 \text { according to the Ten } \\
\text { Group Classification System }\end{array}$ & & 4919 & 95 & $1.9 \%$ \\
\hline
\end{tabular}


TABLE 4 | Continued

\begin{tabular}{|c|c|c|c|c|c|c|c|c|}
\hline $\begin{array}{l}\text { Author } \\
\text { (Ref) }\end{array}$ & $\begin{array}{c}\text { Duration of data } \\
\text { collection }\end{array}$ & City & $\begin{array}{l}\text { Sampling } \\
\text { strategy }\end{array}$ & Population & $\begin{array}{l}\text { Ascertainment } \\
\text { method }\end{array}$ & $\begin{array}{l}\text { Tested } \\
\text { sample }\end{array}$ & $\begin{array}{c}\text { GDM } \\
\text { Positive }\end{array}$ & $\begin{array}{l}\text { Prev. } \\
(\%)\end{array}$ \\
\hline $\begin{array}{l}\text { Masturzo B. } \\
\text { et al. (156) }\end{array}$ & $01 / 2011$ - 12/2015 & Italy, Turin & $\begin{array}{l}\text { Whole } \\
\text { population }\end{array}$ & Singleton pregnancies & Medical records & 27807 & 2308 & $8.3 \%$ \\
\hline $\begin{array}{l}\text { Visconti F. } \\
\text { et al. (157) }\end{array}$ & 08/2011 - 12/2016 & $\begin{array}{l}\text { Italy, } \\
\text { Calabria }\end{array}$ & Consecutive & Singleton pregnancies & IADSPG & 2424 & 596 & $24.7 \%$ \\
\hline $\begin{array}{l}\text { Marozio L. } \\
\text { et al. (158) }\end{array}$ & $2009-2015$ & Italy, Turin & $\begin{array}{l}\text { Whole } \\
\text { population }\end{array}$ & $\begin{array}{l}\text { Pregnant women }<40 \text { years old } \\
\text { Pregnant women between } 40-44 \text { years old } \\
\text { Pregnant women }>45 \text { years old }\end{array}$ & ADA 2014 & $\begin{array}{c}52413 \\
3541 \\
257\end{array}$ & $\begin{array}{c}1430 \\
203 \\
21\end{array}$ & $\begin{array}{l}2.7 \% \\
5.7 \% \\
8.2 \%\end{array}$ \\
\hline Malta & & & & & & & & \\
\hline $\begin{array}{l}\text { Xuereb S. } \\
\text { et al. (159) } \\
\text { Slovenia }\end{array}$ & $01 / 2009-12 / 2009$ & $\begin{array}{l}\text { Malta, } \\
\text { National }\end{array}$ & Consecutive & General population & WHO 2006 & 203 & 43 & $21.2 \%$ \\
\hline $\begin{array}{l}\text { Kek T. et al. } \\
(160) \\
\text { Spain }\end{array}$ & 05/2013 - 09/2015 & $\begin{array}{l}\text { Slovenia, } \\
\text { Ljubljana }\end{array}$ & Unclear & General population & Self-reported & 450 & 43 & $10.0 \%$ \\
\hline $\begin{array}{l}\text { Goni L. } \\
\text { et al. (161) }\end{array}$ & $11 / 2009-03 / 2010$ & $\begin{array}{l}\text { Spain, } \\
\text { Navarra }\end{array}$ & Convenience & General population & Medical records & 5987 & 397 & $7.8 \%$ \\
\hline $\begin{array}{l}\text { Ruiz-Gracia } \\
\text { T. et al. } \\
(162)\end{array}$ & 04/2011 - 03/2012 & $\begin{array}{l}\text { Spain, } \\
\text { Madrid }\end{array}$ & Consecutive & General population & Carpenter-Coustan & 1750 & 185 & $10.5 \%$ \\
\hline $\begin{array}{l}\text { Berglund } \\
\text { SK. et al. } \\
(163)\end{array}$ & $2008-2012$ & $\begin{array}{l}\text { Spain, } \\
\text { Granada }\end{array}$ & Convenience & Overweight and Obese women & $\begin{array}{l}\text { Spanish Society of } \\
\text { Gynecology and } \\
\text { Obstetrics }\end{array}$ & 333 & 46 & $13.8 \%$ \\
\hline $\begin{array}{l}\text { Benaiges D. } \\
\text { et al. (164) }\end{array}$ & $04 / 2013-09 / 2015$ & $\begin{array}{l}\text { Spain, } \\
\text { Barcelona }\end{array}$ & Consecutive & Singleton pregnancies & $\begin{array}{l}\text { National Diabetes } \\
\text { Data Group }\end{array}$ & 1158 & 152 & $13.1 \%$ \\
\hline $\begin{array}{l}\text { Assaf-Balut } \\
\text { C. et al. }\end{array}$ & 01/2015 - 12/2015 & $\begin{array}{l}\text { Spain, } \\
\text { Madrid }\end{array}$ & Consecutive & $\begin{array}{l}\text { Single pregnancy following standard Med-Diet } \\
\text { supplemented with EVOO and pistachios }\end{array}$ & IADSPG & 434 & 74 & $17.1 \%$ \\
\hline$(165)$ & & & & Single pregnancy following standard Med-Diet & & 440 & 74 & $23.4 \%$ \\
\hline $\begin{array}{l}\text { Gortazar L. } \\
\text { et al. (166) }\end{array}$ & $2006-2015$ & $\begin{array}{l}\text { Spain, } \\
\text { Catalonia }\end{array}$ & $\begin{array}{l}\text { Whole } \\
\text { population }\end{array}$ & Singleton pregnancies & Medical records & 739877 & 35729 & $4.8 \%$ \\
\hline $\begin{array}{l}\text { Mane L. } \\
\text { et al. (167) }\end{array}$ & $2010-2013$ & $\begin{array}{l}\text { Spain, } \\
\text { Barcelona }\end{array}$ & $\begin{array}{l}\text { Whole } \\
\text { population }\end{array}$ & General population & Self-reported & 5633 & 572 & $10 \%$ \\
\hline
\end{tabular}

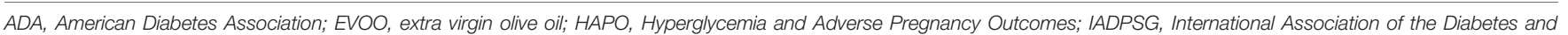
Pregnancy Study Groups; WHO, World Health Organization.

burden of T2DM. Strategies to prevent T2DM may incorporate hyperglycemia screening 4 to 12 weeks after the post-partum as recommended by the most recent guidelines from ADA (12).

Differences in the GDM criteria used in the different countries and sub-regions also play an important role in the differences of prevalence reported and most importantly in the heterogeneity of our meta-analysis estimations. It is known that there is a poor consensus and uniformity in the diagnosis of GDM, as our study demonstrates, by having 24 different criteria used. This fact is to be considered as well with the recent criteria updates, specifically from the WHO in 2013. The differences in GDM criteria allied with the different countries' screening guidelines (e.g., universal GDM screening vs screening for women with risk factors) introduce heterogeneity to the meta-analysis and increases the challenge of comparing the prevalence across countries and regions. Standardized studies and policies across the European region would help to tackle the GDM public health burden.

\section{Strengths, Implications, and Limitations}

This study has used a comprehensive search strategy to review all the studies of GDM in Europe at the regional, sub-regional, and national levels. The study includes a huge number of reports and single estimates that were combined. Estimating a weighted GDM prevalence based on a huge number (over 15 million) of tested pregnant women provides the best-precise estimation of the burden of GDM in the included European countries. Additionally, estimating the pooled GDM prevalence among various pregnant women population groups according to age, trimester of GDM diagnosis, maternal body weight, also provides specific estimates in this population group to priorities action and screening strategies. As mentioned above, the range of GDM per country varied widely therefore we are not able to extrapolate the reported GDM prevalence for the European countries not represented in our estimates, the sub-regions itself and even within the countries, as the case of the Republic of Moldova, Iceland, and Malta that are included in our analysis with one single report. Another potential limitation is the lack of or small number of studies from specific countries which might not reflect the reality of the region. Therefore, interpreting the present findings should be exercised in the light of this important potential limitations.

\section{CONCLUSIONS}

The overall GDM prevalence in Europe is considerable, particularly for pregnant women in Eastern European countries. Epidemiological studies focusing on GDM and using standardized 
TABLE 5 | Weighted national, sub-regional, and regional GDM prevalence in Europe.

\begin{tabular}{|c|c|c|c|c|c|c|c|c|c|c|c|}
\hline \multirow[t]{2}{*}{ Country } & \multirow{2}{*}{$\begin{array}{l}\text { No. of } \\
\text { studies }\end{array}$} & \multirow{2}{*}{$\begin{array}{l}\text { Tested } \\
\text { sample }\end{array}$} & \multirow[t]{2}{*}{ GDM } & \multicolumn{4}{|c|}{ GDM prevalence } & \multicolumn{4}{|c|}{ Heterogeneity measures } \\
\hline & & & & $\begin{array}{c}\text { Range } \\
(\%)\end{array}$ & $\begin{array}{l}\text { Median } \\
(\%)\end{array}$ & $\begin{array}{c}\text { Weighted prev. } \\
\%\end{array}$ & $95 \% \mathrm{Cl}$ & $Q(p-\text { value })^{1}$ & $\begin{array}{c}t^{2} \\
(\%)^{2}\end{array}$ & $\begin{array}{c}95 \% \mathrm{PI} \\
(\%)^{3}\end{array}$ & $\begin{array}{c}P \text {-value } \\
\text { (fixed) }\end{array}$ \\
\hline Eastern Europe & & & & & & & & & & & $\begin{array}{c}p<0.001 \\
(p<0.001)\end{array}$ \\
\hline Hungary & 2 & 10,962 & 1,660 & $10.1-14.9$ & 12.5 & 15.1 & $14.4-15.8$ & - & - & - & \\
\hline Poland & 5 & 1,042 & 298 & $8.0-78.0$ & 13.4 & 34.1 & $8.8-65.8$ & $\begin{array}{c}427.8 \\
(p<0.001)\end{array}$ & 99.1 & $0.00-100$ & \\
\hline $\begin{array}{l}\text { Republic of } \\
\text { Moldova }\end{array}$ & 1 & 118 & 78 & - & - & 66.1 & $57.2-74.0$ & - & - & - & \\
\hline Overall Eastern & 8 & 12,122 & 2,036 & $8.0-78.0$ & 14.2 & 31.5 & $19.8-44.6$ & $\begin{array}{c}665.8 \\
(p<0.001)\end{array}$ & 98.9 & $0.8-79.0$ & \\
\hline Northern Europe & & & & & & & & & & & $\begin{array}{l}p<0.001 \\
(p<0.001)\end{array}$ \\
\hline Denmark & 17 & 474,094 & 19,350 & $0.9-40.1$ & 12.0 & 6.3 & $3.7-9.3$ & $\begin{array}{l}22,782.0 \\
(p<0.001)\end{array}$ & 99.9 & $0.00-24.1$ & \\
\hline Finland & 22 & 749,342 & 129,062 & $4.9-36.3$ & 17.3 & 18.4 & $16.7-20.2$ & $\begin{array}{c}6,728.1 \\
(p<0.001)\end{array}$ & 99.7 & $10.6-27.8$ & \\
\hline Iceland & 3 & 168 & 17 & $2.3-28.9$ & 9.1 & 11.0 & $0.6-29.7$ & $\begin{array}{c}17.5 \\
(p<0.001)\end{array}$ & 88.6 & - & \\
\hline Ireland & 10 & 8,572 & 309 & $1.8-58.4$ & 9.3 & 18.9 & 10.0-29.9 & $\begin{array}{c}376.6 \\
(p<0.001)\end{array}$ & 97.6 & $0.0-64.1$ & \\
\hline Lithuania & 3 & 3,377 & 196 & $2.3-23.6$ & 5.1 & 8.5 & $1.4-20.2$ & $\begin{array}{c}45.1 \\
(p<0.001)\end{array}$ & 95.6 & - & \\
\hline Norway & 19 & 1,332,092 & 25,092 & $1.1-63.0$ & 2.0 & 4.6 & $3.8-5.5$ & $\begin{array}{c}6,094.2 \\
(p<0.001)\end{array}$ & 99.7 & $1.6-8.9$ & \\
\hline Sweden & 20 & $7,479,062$ & 74,073 & $0.2-34.6$ & 1.5 & 1.8 & $1.5-2.2$ & $\begin{array}{l}18,241.0 \\
(p<0.001)\end{array}$ & 99.9 & $0.6-3.8$ & \\
\hline United Kingdom & 28 & 232,214 & 10,113 & $1.9-29.8$ & 11.2 & 11.7 & $9.4-14.4$ & $\begin{array}{c}6,947.8 \\
(p<0.001)\end{array}$ & 99.6 & $1.8-28.6$ & \\
\hline Overall & 122 & $10,278,921$ & 258,212 & $0.2-63.0$ & 7.5 & 8.9 & $7.9-10.0$ & $\begin{array}{l}365,513.4 \\
(p<0.001)\end{array}$ & 100.0 & $1.0-23.4$ & \\
\hline Western Europe & & & & & & & & & & & $\begin{array}{l}p<0.001 \\
(p<0.001)\end{array}$ \\
\hline Austria & 5 & 8,897 & 1,042 & $4.2-46.0$ & 38.3 & 27.3 & $13.0-44.3$ & $\begin{array}{c}796.0 \\
(p<0.001)\end{array}$ & 99.5 & $0.0-90.4$ & \\
\hline Belgium & 2 & 14,773 & 614 & $4.1-11.6$ & 7.9 & 3.9 & $3.6-4.3$ & - & - & - & \\
\hline France & 16 & $3,109,492$ & 189,173 & $1.2-43.8$ & 7.5 & 8.0 & $5.9-10.4$ & $\begin{array}{l}22,936.1 \\
(p<0.001)\end{array}$ & 100.0 & $2.7-17.0$ & \\
\hline Germany & 18 & $1,058,242$ & 101,724 & $3.4-27.6$ & 7.0 & 7.3 & $5.1-9.9$ & $\begin{array}{l}61,693.8 \\
(p<0.001)\end{array}$ & 99.9 & $0.8-21.3$ & \\
\hline Netherlands & 4 & 17,442 & 3,717 & $4.5-31.6$ & 14.0 & 13.9 & $1.9-34.1$ & $\begin{array}{c}2,340.4 \\
(p<0.001)\end{array}$ & 99.9 & $0.0-100.0$ & \\
\hline Switzerland & 10 & 3,877 & 583 & $10.0-31.7$ & 16.1 & 17.0 & $11.3-23.4$ & $\begin{array}{c}120.3 \\
(p<0.001)\end{array}$ & 92.5 & $1.7-41.4$ & \\
\hline Overall Western & 55 & $4,212,723$ & 296,853 & $1.2-46.0$ & 8.6 & 10.7 & $9.5-12.0$ & $\begin{array}{l}73,483.9 \\
(p<0.001)\end{array}$ & 99.9 & $3.4-21.4$ & \\
\hline Southern Europe & & & & & & & & & & & $\begin{array}{c}p<0.001 \\
(p<0.001)\end{array}$ \\
\hline Croatia & 13 & 88,086 & 4,676 & $1.1-23.1$ & 4.7 & 5.8 & $3.2-9.2$ & $\begin{array}{c}3,635.5 \\
(p<0.001)\end{array}$ & 99.7 & $0.0-24.0$ & \\
\hline Cyprus & 1 & 230 & 45 & - & - & 19.6 & $15.0-25.2$ & - & - & - & \\
\hline Greece & 4 & 1,122 & 102 & $7.6-17.0$ & 9.3 & 10.0 & $6.4-14.3$ & $\begin{array}{c}69.6 \\
(p=0.02)\end{array}$ & 9.9 & $0.1-31.3$ & \\
\hline Italy & 32 & 222,809 & 13,497 & $1.7-47.6$ & 11.5 & 14.5 & $11.1-18.1$ & $\begin{array}{l}13,663.2 \\
(p<0.001)\end{array}$ & 99.8 & $0.9-39.8$ & \\
\hline Malta & 1 & 203 & 43 & - & - & 21.2 & $16.1-27.3$ & - & - & - & \\
\hline Slovenia & 1 & 450 & 43 & - & - & 9.6 & $7.2-12.6$ & - & - & - & \\
\hline Spain & 17 & 756,181 & 37,786 & $4.8-39.6$ & 11.4 & 15.0 & $11.0-19.4$ & $\begin{array}{c}1,838.4 \\
(p<0.001)\end{array}$ & 99.1 & $1.7-37.6$ & \\
\hline Overall Southern & 69 & $1,069,081$ & 56,192 & $1.1-47.6$ & 10.7 & 12.3 & $10.9-13.9$ & $\begin{array}{l}19,346.8 \\
(p<0.001)\end{array}$ & 99.6 & $3.0-28.0$ & \\
\hline
\end{tabular}


TABLE 5 | Continued

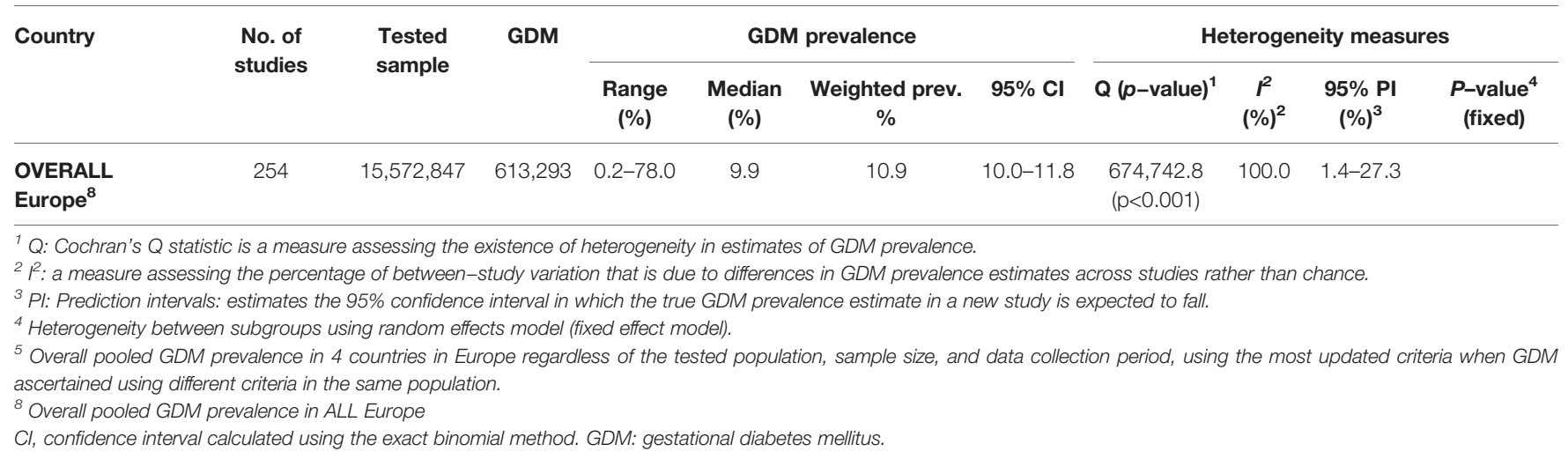

Risk of Bias assessment

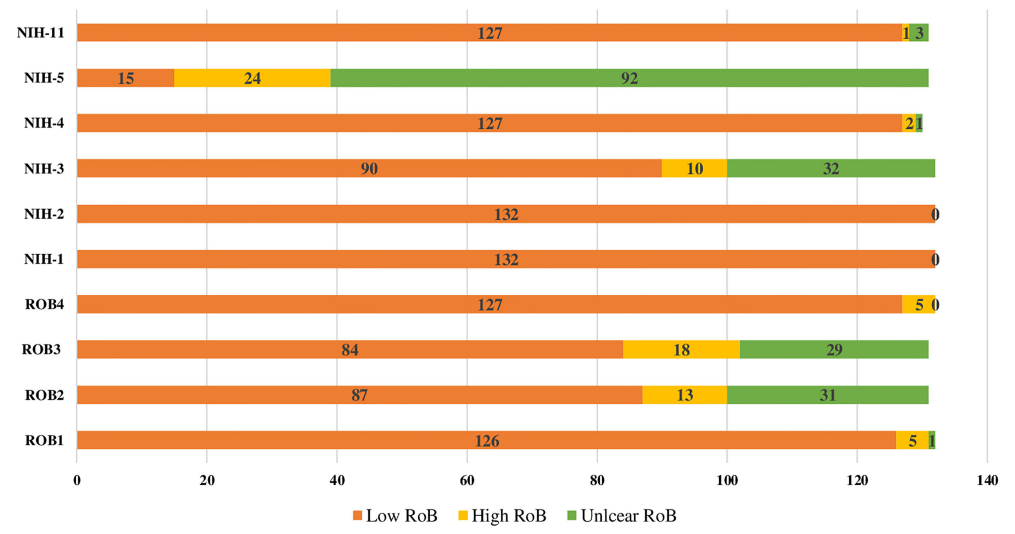

FIGURE 2 | Risk of Bias assesment of the 132 reviewed research reports on GDM. RoB1: GDM ascertainment (1: biological assay/medical records; 2: selfreported; 3: unclear) RoB2: Sampling methodology (1: probability-based "random, consecutive, or whole population within a specified period of time" ; 2: nonprobability based; 3: unclear) RoB3: Response rate (1:<80\%; 2:80\%) RoB4: Precision (1: tested sample size100; 2: tested sample size <100) NIH-1: Was the research question or objective in this paper clearly stated? 1: Low risk of bias (ROB), 2: High ROB, 3: Unclear ROB NIH-2: Was the study population clearly specified and defined? 1: Low ROB, 2: High ROB, 3: Unclear ROB NIH-3: Was the participation rate of eligible persons at least 50\%? 1: Low ROB, 2: High ROB, 3: Unclear ROB NIH-3: Was the participation rate of eligible persons at least 50\%? 1: Low ROB, 2: High ROB, 3:Unclear ROB prespecified and applied uniformly to ail participants? 1: Low ROB, 2: High ROB, 3: Unclear ROB NIH-5: Was a sample size justification, power description, or variance and effect estimates provided? 1: Low ROB, 2: High ROB, 3: Unclear ROB NIH-11: Were the outcome measures (dependent variables) clearly defined, valid, reliable, and implemented consistently across all study participants? 1: Low ROB, 2: High ROB, 3: Unclear ROB.

GDM criteria would be crucial to better estimate the national, subregional, and regional GDM of Europe as GDM has serious public health implications for the life of the mothers and newborns. This systematic review and meta-analysis findings highlight these implications and aim to contribute to the vigilant public health awareness campaigns about the risk factors associated with developing GDM in Europe and globally.

\section{DATA AVAILABILITY STATEMENT}

The original contributions presented in the study are included in the article/Supplementary Material. Further inquiries can be directed to the corresponding author.

\section{ETHICS STATEMENT}

There are no primary data used in this review. There is no need for any ethical approval or an exemption letter according to the United Arab Emirates University-Human Research Ethics Committee.

\section{AUTHOR CONTRIBUTIONS}

RHA conceptualized and designed the study. MSP assessed the eligibility of the retrieved citations in the titles/abstracts and fulltext screening phases. RHA, NA, and MSP critically assessed the eligible studies and extracted data. RHA and NA performed the 
analysis. MSP and RB-S wrote the initial draft of the manuscript. All authors contributed to the article and approved the submitted version.

\section{FUNDING}

This systematic review was funded by the Summer Undergraduate Research Experience (SURE) PLUS-Grant of the United Arab Emirates University, 2017 (Research grant: 31M348). The funder

\section{REFERENCES}

1. Duke L, Fereira de Moura A, de Lapertosa S, Hammond L, Jacobs E, Kaundal A, et al. IDF Diabetes Atlas 9th edition 2019. Int Diabetes Fed Diabetes Atlas, Ninth Ed. (2019). Available at: https://www.diabetesatlas.org/ en/ [Accessed February 21, 2021].

2. World Health Organization. Diagnostic Criteria and Classification of Hyperglycaemia First Detected in Pregnancy. Geneva, Switzerland: WHO (2013). Available at: https://apps.who.int/iris/handle/10665/85975 [Accessed February 21, 2021].

3. Metzger BE, Gabbe SG, Persson B, Lowe LP, Dyer AR, Oats JJN, et al. International Association of Diabetes and Pregnancy Study Groups Recommendations on the Diagnosis and Classification of Hyperglycemia in Pregnancy. Diabetes Care Am Diabetes Assoc (2010) 33:e98-8. Response to Weinert. doi: $10.2337 / \mathrm{dc} 10-0719$

4. Association AD. 2. Classification and Diagnosis of Diabetes: Standards of Medical Care in Diabetes-2021. Diabetes Care (2021) 44(Supplement 1): S15-33. doi: $10.2337 / \mathrm{dc} 21-S 002$

5. Di Cesare M, Bentham J, Stevens GA, Zhou B, Danaei G, Lu Y, et al. Trends in Adult Body-Mass Index in 200 Countries From 1975 to 2014: A Pooled Analysis of 1698 Population-Based Measurement Studies With 19.2 Million Participants. Lancet (2016) 387(10026):1377-96. doi: 10.1016/S0140-6736 (16)30054-X

6. WHO Regional Office for Europe. Health for All Explorer - European Health Information Gateway (2018). Available at: https://gateway.euro.who.int/en/ hfa-explorer/\#1iMRgJreZp.

7. Pineda E, Sanchez-Romero LM, Brown M, Jaccard A, Jewell J, Galea G, et al. Forecasting Future Trends in Obesity Across Europe: The Value of Improving Surveillance. Obes Facts (2018) 11(5):360-71. doi: 10.1159/ 000492115

8. Janssen F, Bardoutsos A, Vidra N. Obesity Prevalence in the Long-Term Future in 18 European Countries and in the USA. Obes Facts (2020) 13 (5):514-27. doi: 10.1159/000511023

9. Lutsiv O, Mah J, Beyene J, Mcdonald SD. The Effects of Morbid Obesity on Maternal and Neonatal Health Outcomes: A Systematic Review and MetaAnalyses. Obes Rev (2015) 16(7):531-46. doi: 10.1111/obr.12283

10. David McIntyre H, Jensen DM, Jensen RC, Kyhl HB, Jensen TK, Glintborg D, et al. Gestational Diabetes Mellitus: Does One Size Fit All? A Challenge to Uniform Worldwide Diagnostic Thresholds. Diabetes Care (2018) 41 (7):1339-42. doi: 10.2337/dc17-2393

11. European Commission. Women Are Having Their First Child at an Older Age [Internet]. Products Eurostat News - Eurostat (2020). Available at: https://ec. europa.eu/eurostat/web/products-eurostat-news/-/DDN-20200515-2.

12. American Diabetes Association. Management of Diabetes in Pregnancy: Standards of Medical Care in Diabetes-2021. Diabetes Care (2021) 44 (Supplement 1):S200-10. doi: 10.2337/dc21-S014

13. Metzger BE, Lowe LP, Dyer AR, Trimble ER, Sheridan B, Hod M, et al. Hyperglycemia and Adverse Pregnancy Outcome (HAPO) Study: Associations With Neonatal Anthropometrics. Diabetes (2009) 58(2):4539. doi: $10.2337 / \mathrm{db} 08-1112$

14. Dabelea D. The Predisposition to Obesity and Diabetes in Offspring of Diabetic Mothers. Diabetes Care (2007) 30:S169-74. doi: 10.2337/dc07-s211 had no role in the study design, collection, analysis, or interpretation of the data, nor in writing and the decision to submit this article for publication.

\section{SUPPLEMENTARY MATERIAL}

The Supplementary Material for this article can be found online at: https://www.frontiersin.org/articles/10.3389/fendo.2021. 691033/full\#supplementary-material

15. Lowe WL, Scholtens DM, Lowe LP, Kuang A, Nodzenski M, Talbot O, et al Association of Gestational Diabetes With Maternal Disorders of Glucose Metabolism and Childhood Adiposity. JAMA J Am Med Assoc (2018) 320 (10):1005-16. doi: 10.1001/jama.2018.11628

16. World Health Organization. WHO/Europe | Diabetes Epidemic in Europe (2011). Available at: https://www.euro.who.int/en/health-topics/ noncommunicable-diseases/diabetes/news/news/2011/11/diabetesepidemic-in-europe.

17. Bellamy L, Casas JP, Hingorani AD, Williams D. Type 2 Diabetes Mellitus After Gestational Diabetes: A Systematic Review and Meta-Analysis. Lancet (2009) 373(9677):1773-9. doi: 10.1016/S0140-6736(09)60731-5

18. Wah Cheung N, Moses RG. Gestational Diabetes Mellitus: Is it Time to Reconsider the Diagnostic Criteria? Diabetes Care (2018) 41:1337-8. doi: $10.2337 / \mathrm{dci} 18-0013$

19. Hod M, Kapur A, McIntyre HD. Evidence in Support of the International Association of Diabetes in Pregnancy Study Groups' Criteria for Diagnosing Gestational Diabetes Mellitus Worldwide in 2019. Am J Obstet Gynecol (2019) 221(2):109-16. doi: 10.1016/j.ajog.2019.01.206

20. Egan AM, Vellinga A, Harreiter J, Simmons D, Desoye G, Corcoy R, et al. Epidemiology of Gestational Diabetes Mellitus According to IADPSG/WHO 2013 Criteria Among Obese Pregnant Women in Europe. Diabetologia (2017) 60(10):1913-21. doi: 10.1007/s00125-017-4353-9

21. Stevens GA, Alkema L, Black RE, Boerma JT, Collins GS, Ezzati M, et al. Guidelines for Accurate and Transparent Health Estimates Reporting: The GATHER Statement. PloS Med (2016) 13(6):e1002056. doi: 10.1371/ journal.pmed.1002056

22. Eades CE, Cameron DM, Evans JMM. Prevalence of Gestational Diabetes Mellitus in Europe: A Meta-Analysis. Diabetes Res Clin Pract (2017) 129:173-81. doi: 10.1016/j.diabres.2017.03.030

23. Liberati A, Altman DG, Tetzlaff J, Mulrow C, Gøtzsche PC, Ioannidis JPA, et al. The PRISMA Statement for Reporting Systematic Reviews and MetaAnalyses of Studies That Evaluate Health Care Interventions: Explanation and Elaboration. PloS Med (2009) 6(7):e1000100. doi: 10.1371/ journal.pmed.1000100

24. Al-Rifai RH, Abdo NM, Paulo MS, Saba S, Ahmed L. Prevalence of Gestational Diabetes Mellitus in the Middle East and North Africa, 20002019: A Systematic Review, Meta-Analysis, and Meta-Regression. Front Endocrinol (Lausanne) (2021) 12. doi: 10.3389/fendo.2021.668447

25. United Nations Statistics Division. Methdoology - Standard Country or Area Codes for Statistical Use. New York, USA: Statistics Division United Nations (1999).

26. Babineau J. Product Review: Covidence (Systematic Review Software). JCHLA / JABSC (2014) 35(2):68-71. doi: 10.5596/c14-016

27. Covidence. Covidence - Better Systematic Review Management. Available at: https://www.covidence.org/home.

28. Freeman MF, Tukey JW. Transformations Related to the Angular and the Square Root. Ann Math Stat (1950) 21(4):607-11. doi: 10.1214/aoms/ 1177729756

29. Miller JJ. The Inverse of the Freeman-Tukey Double Arcsine Transformation. Am Stat (1978) 32(4):138. doi: 10.1080/00031305.1978.10479283

30. DerSimonian R, Laird N. Meta-Analysis in Clinical Trials Revisited. Contemp Clin Trials (2015) 45(Pt A):139-45. doi: 10.1016/j.cct.2015.09.002 
31. Borenstein M, Rothstein H, Hedges L, Higgins J. Introduction to MetaAnalysis. West Sussex, United Kingdom: John Wiley \& Sons, Ltd (2011). 450 p. doi: 10.1002/9780470743386.fmatter

32. National Heart Lung and Blood Institute. Study Quality Assessment Tools NHLBI. NIH (2016). Available at: https://www.nhlbi.nih.gov/health-topics/ study-quality-assessment-tools [Accessed November 21, 2018].

33. Sterne JAC, Egger M. Funnel Plots for Detecting Bias in Meta-Analysis: Guidelines on Choice of Axis. J Clin Epidemiol (2001) 54(10):1046-55. doi: 10.1016/S0895-4356(01)00377-8

34. Nyaga VN, Arbyn M, Aerts M. Metaprop: A Stata Command to Perform Meta-Analysis of Binomial Data. Arch Public Heal (2014) 72(1):1-10. doi: 10.1186/2049-3258-72-39

35. StataCorp. Stata Statistical Software: Realease 16. Texas, USA: StataCorp (2017).

36. Rénes L, Barka N, Gyurkovits Z, Paulik E, Németh G, Orvos H. The Journal of Maternal-Fetal \& Neonatal Medicine Predictors of Caesarean Section-a Cross-Sectional Study in Hungary. J Matern Neonatal Med (2018) 1-8. doi: 10.1080/14767058.2017.1285888

37. Kun A, Szabó E, Tornoczky J, Kerenyi Z, Tabák ÁG. Increasing Prevalence of Gestational Diabetes According to the Results of a Population-Based Screening Programme in Hungary Between 2009-2017. (2018). Available at: https://www.easd.org/virtualmeeting/home.html\#!resources/increasingprevalence-of-gestational-diabetes-according-to-the-results-of-apopulation-based-screening-programme-in-hungary-between-2009-2017ab604c0c-d02c-4fbe-8475-146eefdfdc23 [Accessed April 28, 2020].

38. Mac-Marcjanek K, Zieleniak A, Wozniak L, Zurawska-Klis M, Cypryk K, Okla P, et al. Comparison of Leukocyte IL6 Expression in Patients With Gestational Diabetes Mellitus (GDM) Diagnosed by the Polish Diabetes Association (PDA) 2011 and 2014 Criteria. Endokrynol Pol (2017) 68 (3):317-25. doi: 10.5603/EP.a2017.0014

39. Kosinska-Kaczynska K, Szymusik I, Kaczynski B, Wielgos M. Observational Study of Associations Between Gestational Weight Gain and Perinatal Outcomes in Dichorionic Twin Pregnancies. Int J Gynecol Obstet (2017) 138(1):94-9. doi: 10.1002/ijgo.12171

40. Szymusik I, Kosinski P, Kosinska-Kaczynska K, Warzecha D, Karwacka A, Kaczynski B, et al. Academy's Paper The First Trimester Aneuploidy Biochemical Markers in IVF/ICSI Patients Have No Additional Benefit Compared to Spontaneous Conceptions in the Prediction of Pregnancy Complications. J Perinat Med (2018) 46(9):953-9. doi: 10.1515/jpm-20170199

41. Brankica K, Valentina VN, Slagjana SK, Sasha JM. Maternal 75-G OGTT Glucose Levels as Predictive Factors for Large-for-Gestational Age Newborns in Women With Gestational Diabetes Mellitus. Arch Endocrinol Metab (2016) 60(1):36-41. doi: 10.1590/2359-3997000000126

42. Bonnesen B, Oddgeirsdóttir HL, Naver KV, Jørgensen FS, Nilas L. Women With Minor Menstrual Irregularities Have Increased Risk of Preeclampsia and Low Birthweight in Spontaneous Pregnancies. Acta Obstet Gynecol Scand (2016) 95(1):88-92. doi: 10.1111/aogs.12792

43. Medek H, Halldorsson T, Gunnarsdottir I, Geirsson RT. Physical Activity of Relatively High Intensity in Mid-Pregnancy Predicts Lower Glucose Tolerance Levels. Acta Obstet Gynecol Scand (2016) 95(9):1055-62. doi: 10.1111/aogs.12931

44. Holst S, Kjær SK, Jørgensen ME, Damm P, Jensen A. Fertility Problems and Risk of Gestational Diabetes Mellitus: A Nationwide Cohort Study. Fertil Steril (2016) 106(2):427-434.e1. doi: 10.1016/j.fertnstert.2016.03.040

45. Jeppesen C, Maindal HT, Kristensen JK, Ovesen PG, Witte DR. National Study of the Prevalence of Gestational Diabetes Mellitus Among Danish Women From 2004 to 2012. Scand J Public Health (2017) 45(8):811-7. doi: 10.1177/1403494817736943

46. Hamann CR, Egeberg A, Wollenberg A, Gislason G, Skov L, Thyssen JP. Pregnancy Complications, Treatment Characteristics and Birth Outcomes in Women With Atopic Dermatitis in Denmark. J Eur Acad Dermatol Venereol (2019) 33(3):577-87. doi: 10.1111/jdv.15256

47. Strand-Holm KM, Fuglsang J, Ovesen PG, Maimburg RD. Diabetes Mellitus and Lower Genital Tract Tears After Vaginal Birth: A Cohort Study. Midwifery (2019) 69:121-7. doi: 10.1016/j.midw.2018.11.010

48. Koivusalo SB, Rönö K, Klemetti MM, Roine RP, Lindström J, Erkkola M, et al. Gestational Diabetes Mellitus Can Be Prevented by Lifestyle
Intervention: The Finnish Gestational Diabetes Prevention Study (RADIEL): A Randomized Controlled Trial. Diabetes Care (2016) 39 (1):24-30. doi: 10.2337/dc15-0511

49. Ellenberg A, Sarvilinna N, Gissler M, Ulander V-M. New Guidelines for Screening, Diagnosing, and Treating Gestational Diabetes - Evaluation of Maternal and Neonatal Outcomes in Finland From 2006 to 2012. Acta Obstet Gynecol Scand (2017) 96(3):372-81. doi: 10.1111/aogs.13074

50. Koivunen S, Torkki A, Bloigu A, Gissler M, Pouta A, Kajantie E, et al. Towards National Comprehensive Gestational Diabetes Screening Consequences for Neonatal Outcome and Care. Acta Obstet Gynecol Scand (2017) 96(1):106-13. doi: 10.1111/aogs.13030

51. Meinilä J, Valkama A, Koivusalo SB, Stach-Lempinen B, Rönö K, Lindström J, et al. Is Improvement in the Healthy Food Intake Index (HFII) Related to a Lower Risk for Gestational Diabetes? Br J Nutr (2017) 117(8):1103-9. doi: 10.1017/S0007114517001015

52. Laine MK, Kautiainen H, Gissler M, Raina M, Aahos I, Järvinen K, et al. Gestational Diabetes in Primiparous Women-Impact of Age and Adiposity: A Register-Based Cohort Study. Acta Obstet Gynecol Scand (2018) 97 (2):187-94. doi: 10.1111/aogs.13271

53. Laine MK, Kautiainen H, Gissler M, Raina M, Aahos I, Järvinen K, et al. Short Primiparous Women are at an Increased Risk for Gestational Diabetes Mellitus. Public Health (2018) 156:101-8. doi: 10.1016/j.puhe.2017.12.020

54. Girchenko P, Tuovinen S, Lahti-Pulkkinen M, Lahti J, Savolainen K, Heinonen K, et al. Maternal Early Pregnancy Obesity and Related Pregnancy and Pre-Pregnancy Disorders: Associations With Child Developmental Milestones in the Prospective PREDO Study. Int J Obes (2018) 42(5):995-1007. doi: 10.1038/s41366-018-0061-x

55. Kong L, Nilsson IAK, Gissler M, Lavebratt C. Associations of Maternal Diabetes and Body Mass Index With Offspring Birth Weight and Prematurity Supplemental Content. JAMA Pediatr (2019) 173(4):371-8. doi: 10.1001/jamapediatrics.2018.5541

56. Ellfolk M, Leinonen MK, Gissler M, Lahesmaa-Korpinen A-M, Saastamoinen L, Nurminen M-L, et al. Second-Generation Antipsychotics and Pregnancy Complications. Eur J Clin Pharmacol (2020) 76:107-15. doi: 10.1007/s00228-019-02769-z

57. Ijäs H, Koivunen S, Raudaskoski T, Kajantie E, Gissler M, Vääräsmäki M. Independent and Concomitant Associations of Gestational Diabetes and Maternal Obesity to Perinatal Outcome: A Register-Based Study. PloS One (2019) 14(8):1-11. doi: 10.1371/journal.pone.0221549

58. Tryggvadottir EA, Medek H, Geirsson RT, Gunnarsdottir I. Association Between Healthy Maternal Dietary Pattern and Risk for Gestational Diabetes Mellitus. Eur J Clin Nutr Adv Online Publ (2015) 70:237-42. doi: 10.1038/ ejcn.2015.145

59. Lindsay KL, Kennelly M, Culliton M, Smith T, Maguire OC, Shanahan F, et al. Probiotics in Obese Pregnancy do Not Reduce Maternal Fasting Glucose: A Double-Blind, Placebo-Controlled, Randomized Trial (Probiotics in Pregnancy Study). Am J Clin Nutr (2014) 99(6):1432-9. doi: 10.3945/ajcn.113.079723

60. Daly N, Stapleton M, O’Kelly R, Kinsley B, Daly S, Turner MJ. The Role of Preanalytical Glycolysis in the Diagnosis of Gestational Diabetes Mellitus in Obese Women. Am J Obstet Gynecol (2015) 213(1):84.e1-5. doi: 10.1016/ j.ajog.2015.03.022

61. Mone F, Adams B, Manderson JG, Mcauliffe FM. The East Timorese: A High-Risk Ethnic Minority in UK Obstetrics: A Cohort Study. J Matern Neonatal Med (2015) 28(13):1594-7. doi: 10.3109/14767058.2014.962507

62. Moore R, Adler H, Jackson V, Lawless M, Byrne M, Eogan M, et al. Impaired Glucose Metabolism in HIV-Infected Pregnant Women: A Retrospective Analysis. Int J STD AIDS (2016) 27(7):581-5. doi: 10.1177/0956462415587625

63. O'dea A, Tierney M, Danyliv A, Glynn LG, Mcguire BE, Carmody LA, et al. Screening for Gestational Diabetes Mellitus in Primary Versus Secondary Care: The Clinical Outcomes of a Randomised Controlled Trial. Diabetes Res Clin Pract (2016) 117:55-63. doi: 10.1016/j.diabres.2016.04.0230168-8227/Ó

64. Farren M, Daly N, McKeating A, Kinsley B, Turner MJ, Daly S. The Prevention of Gestational Diabetes Mellitus With Antenatal Oral Inositol Supplementation: A Randomized Controlled Trial. Diabetes Care (2017) 40 (6):759-63. doi: 10.2337/dc16-2449

65. Daly N, Farren M, McKeating A, O’Kelly R, Stapleton M, Turner MJ. A Medically Supervised Pregnancy Exercise Intervention in Obese Women: A 
Randomized Controlled Trial. Obstet Gynecol (2017) 130(5):1001-10. doi: 10.1097/AOG.0000000000002267

66. Ramonienė G, Maleckienė L, Nadišauskienė RJ, Bartusevičienẻ E, Railaite DR, Mačiulevičienè R, et al. Maternal Obesity and Obstetric Outcomes in a Tertiary Referral Center. Med (2017) 53(2):109-13. doi: 10.1016/ j.medici.2017.03.003

67. Malakauskienė L, Nadišauskienė RJ, Ramašauskaite D, Bartusevičienė E, Ramoniené G, Maleckienè L. Is it Necessary to Postpone Pregnancy After Bariatric Surgery: A National Cohort Study. J Obstet Gynaecol (Lahore) [Internet] (2019) 0(0):1-5. doi: 10.1080/01443615.2019.1634024

68. Rasmussen S, Irgens L, Espinoza J. Maternal Obesity and Excess of Fetal Growth in Pre-Eclampsia. BJOG Int J Obstet Gynaecol (2014) 121(11):13518. doi: 10.1111/1471-0528.12677

69. Sommer C, Mørkrid K, Jenum AK, Sletner L, Mosdøl A, Birkeland KI. Weight Gain, Total Fat Gain and Regional Fat Gain During Pregnancy and the Association With Gestational Diabetes: A Population-Based Cohort Study. Int J Obes (2013) 38:76-81. doi: 10.1038/ijo.2013.185

70. Helseth R, Salvesen O, Stafne SN, Morkved S, Salvesen KA, Carlsen SM. Gestational Diabetes Mellitus Among Nordic Caucasian Women: Prevalence and Risk Factors According to WHO and Simplified IADPSG Criteria. Scand J Clin Lab Invest (2014) 74(7):620-8. doi: 10.3109/ 00365513.2014.928942

71. Leirgul E, Brodwall K, Greve G, Vollset SE, Holmstrøm H, Tell GS, et al. Maternal Diabetes, Birth Weight, and Neonatal Risk of Congenital Heart Defects in Norway, 1994-2009. Obstet Gynecol (2016) 128(5):1116-25. doi: 10.1097/AOG.0000000000001694

72. Garnæs KK, Mørkved S, Salvesen $\varnothing$, Moholdt T. Exercise Training and Weight Gain in Obese Pregnant Women: A Randomized Controlled Trial (ETIP Trial). PloS Med (2016) 13(7):1-18. doi: 10.1371/ journal.pmed.1002079

73. Sorbye LM, Skjaerven R, Klungsoyr K, Morken NH. Gestational Diabetes Mellitus and Interpregnancy Weight Change: A Population-Based Cohort Study. PloS Med (2017) 14(8):1-11. doi: 10.1371/journal.pmed.1002367

74. Lehmann S, Baghestan E, Børdahl PE, Muller Irgens L, Rasmussen SA. Trial of Labor After Cesarean Section in Risk Pregnancies: A Population-Based Cohort Study. Acta Obstet Gynecol Scand (2019) 98(7):894-904. doi: 10.1111/aogs.13565

75. Sole KB, Staff AC, Laine K. The Association of Maternal Country of Birth and Education With Hypertensive Disorders of Pregnancy: A PopulationBased Study of 960516 Deliveries in Norway. Acta Obstet Gynecol Scand (2018) 97(10):1237-47. doi: 10.1111/aogs.13393

76. Magnus MC, Wilcox AJ, Morken N-H, Weinberg CR, Håberg SE. Role of Maternal Age and Pregnancy History in Risk of Miscarriage: Prospective Register Based Study. BMJ (2019) 364:869. doi: 10.1136/bmj.1869

77. Lindqvist M, Persson M, Lindkvist M, Mogren I. No Consensus on Gestational Diabetes Mellitus Screening Regimes in Sweden: Pregnancy Outcomes in Relation to Different Screening Regimes 2011 to 2012, a CrossSectional Study. BMC Pregnancy Childbirth (2014) 14(1):185. doi: 10.1186/ 1471-2393-14-185

78. Nilsson C, Andersson C, Ursing D, Strevens H, Landin-Olsson M. Outcomes of Women With Gestational Diabetes Mellitus in Sweden. Eur J Obstet Gynecol Reprod Biol (2015) 193:132-5. doi: 10.1016/j.ejogrb.2015.07.021

79. Stokkeland K, Ludvigsson JF, Hultcrantz R, Ekbom A, Höijer J, Bottai M, et al. Increased Risk of Preterm Birth in Women With Autoimmune Hepatitis - A Nationwide Cohort Study. Liver Int (2016) 36(1):76-83. doi: $10.1111 /$ liv.12901

80. Sundelin HEK, Stephansson O, Hultman CM, Ludvigsson JF. Pregnancy Outcomes in Women With Autism: A Nationwide Population-Based Cohort Study. Clin Epidemiol (2018) 10:1817-26. doi: 10.2147/CLEP.S176910

81. Stogianni A, Lendahls L, Landin-Olsson M, Thunander M. Obstetric and Perinatal Outcomes in Pregnancies Complicated by Diabetes, and Control Pregnancies, in Kronoberg, Sweden. BMC Pregnancy Childbirth (2019) 19 (1):159. doi: 10.1186/s12884-019-2269-8

82. Crump C, Sundquist J, Winkleby MA, Sundquist K. Preterm Birth and Risk of Chronic Kidney Disease From Childhood Into Mid-Adulthood: National Cohort Study. BMJ (2019) 365:1-8. doi: 10.1136/bmj.11346

83. Hildén K, Hanson U, Persson M, Magnuson A, Simmons D, Fadl H. Gestational Diabetes and Adiposity Are Independent Risk Factors for
Perinatal Outcomes: A Population Based Cohort Study in Sweden. Diabetes Med (2019) 36(2):151-7. doi: 10.1111/dme.13843

84. Khashan AS, Evans M, Kublickas M, McCarthy FP, Kenny LC, Stenvinkel P, et al. Erratum: Preeclampsia and Risk of End Stage Kidney Disease: A Swedish Nationwide Cohort Study. PloS Med (2019) 16(7):e1002875. doi: 10.1371/journal.pmed.1002875

85. Liu C, Ahlberg M, Hjern A, Stephansson O. Perinatal Health of Refugee and Asylum-Seeking Women in Sweden 2014-17: A Register-Based Cohort Study. Eur J Public Health (2019) 29(6):1048-55. doi: 10.1093/eurpub/ ckz120

86. Farrar D, Fairley L, Wright J, Tuffnell D, Whitelaw D, Lawlor DA. Evaluation of the Impact of Universal Testing for Gestational Diabetes Mellitus on Maternal and Neonatal Health Outcomes: A Retrospective Analysis. BMC Pregnancy Childbirth (2014) 14(1):1-8. doi: 10.1186/14712393-14-317

87. West J, Lawlor D, Fairley L, Wright J. Differences in Socioeconomic Position, Lifestyle and Health-Related Pregnancy Characteristics Between Pakistani and White British Women in the Born in Bradford Prospective Cohort Study: The Influence of the Woman's, Her Partner's and Their Parents' Place of Birth. BMJ Open (2014) 4:1-12. doi: 10.1136/bmjopen-2014-004805

88. Syngelaki A, Pastides A, Kotecha R, Wright A, Akolekar R, Nicolaides KH. First-Trimester Screening for Gestational Diabetes Mellitus Based on Maternal Characteristics and History. Fetal Diagn Ther (2015) 38(1):1421. doi: $10.1159 / 000369970$

89. Thomas S, Poston UL, Pasupathy D, Patel MBBS N, Sandall J, Seed CStat PT, et al. Eff Ect of a Behavioural Intervention in Obese Pregnant Women (the UPBEAT Study): A Multicentre, Randomised Controlled Trial. Lancet Diabetes Endocrinol (2015) 3:767-77. doi: 10.1016/S2213-8587(15)00227-2

90. Sovio U, Murphy HR, Smith GCS. Accelerated Fetal Growth Prior to Diagnosis of Gestational Diabetes Mellitus: A Prospective Cohort Study of Nulliparous Women. Diabetes Care (2016) 39:982-7. doi: 10.2337/dc160160

91. Murphy A, NA B. Compliance With National Institute of Health and Care Excellence Risk-Based Screening for Gestational Diabetes Mellitus in Nulliparous Women. Item Type Article Risk-Based Screening for Gestational Diabetes Mellitus in Nulliparous Women. Eur J Obs Gynecol Reprod Biol (2016) 199:60-5. doi: 10.1016/j.ejogrb.2016.01.044

92. White SL, Lawlor DA, Briley AL, Godfrey KM, Nelson SM, Oteng-Ntim E, et al. Early Antenatal Prediction of Gestational Diabetes in Obese Women: Development of Prediction Tools for Targeted Intervention. PloS One (2016) 11(12):1-17. doi: 10.1371/journal.pone.0167846

93. Hanna FW, Duff CJ, Shelley-Hitchen A, Hodgson E, Fryer AA. Diagnosing Gestational Diabetes Mellitus: Implications of Recent Changes in Diagnostic Criteria and Role of Glycated Haemoglobin (HbAlc). Clin Med J R Coll Physicians London (2017) 17(2):108-13. doi: 10.7861/ clinmedicine.17-2-108

94. Panaitescu AM, Syngelaki A, Prodan N, Akolekar R, Nicolaides KH. Chronic Hypertension and Adverse Pregnancy Outcome: A Cohort Study. Ultrasound Obstet Gynecol (2017) 50(2):228-35. doi: 10.1002/uog.17493

95. Hall E, Franklin M, Rajasingam D, Chandiramani M, Reid A, Atkinson R, et al. Introducing a New Model of Care for Women With Gestational Diabetes. Diabet Med (2018) 35(Supplement 1):174.

96. Balani J, Hyer S, Shehata H, Mohareb F. Visceral Fat Mass as a Novel Risk Factor for Predicting Gestational Diabetes in Obese Pregnant Women. Obstet Med (2018) 11(3):121-5. doi: 10.1177/1753495X17754149

97. Nzelu D, Dumitrascu-Biris D, Hunt KF, Cordina M, Kametas NA. Pregnancy Outcomes in Women With Previous Gestational Hypertension: A Cohort Study to Guide Counselling and Management. Pregnancy Hypertens (2018) 12:194-200. doi: 10.1016/j.preghy.2017.10.011

98. Vieira MC, Begum S, Seed PT, Badran D, Briley AL, Gill C, et al. Gestational Diabetes Modifies the Association Between PlGF in Early Pregnancy and Preeclampsia in Women With Obesity. Pregnancy Hypertens (2018) 13 (June):267-72. doi: 10.1016/j.preghy.2018.07.003

99. Wagnild JM, Hinshaw K, Pollard TM. Associations of Sedentary Time and Self-Reported Television Time During Pregnancy With Incident Gestational Diabetes and Plasma Glucose Levels in Women at Risk of Gestational Diabetes in the UK. BMC Public Health (2019) 19(1):1-8. doi: 10.1186/ s12889-019-6928-5 
100. Bozkurt L, Göbl CS, Pfligl L, Leitner K, Bancher-Todesca D, Luger A, et al. Pathophysiological Characteristics and Effects of Obesity in Women With Early and Late Manifestation of Gestational Diabetes Diagnosed by the International Association of Diabetes and Pregnancy Study Groups Criteria. J Clin Endocrinol Metab (2015) 100(3):1113-20. doi: 10.1210/jc.2014-4055

101. Tramontana A, Dieplinger B, Stangl G, Hafner E, Dieplinger H. First Trimester Serum Afamin Concentrations Are Associated With the Development of Pre-Eclampsia and Gestational Diabetes Mellitus in Pregnant Women. Clin Chim Acta (2018) 476:160-6. doi: 10.1016/ j.cca.2017.11.031

102. Tramontana A, Pablik E, Stangl G, Hartmann B, Dieplinger H, Hafner E. Accepted Manuscript Combination of First Trimester Serum Afamin Levels and Three-Dimensional Placental Bed Vascularization as a Possible Screening Method to Detect Women at-Risk for Adverse Pregnancy Complications Like Pre-Eclampsia and Gestational Diabetes Mellitus in Low-Risk Pregnancies. Placenta (2017) 62:9-15. doi: 10.1016/ j.placenta.2017.12.014

103. Köninger A, Iannaccone A, Hajder E, Frank M, Schmidt B, Schleussner E, et al. Afamin Predicts Gestational Diabetes in Polycystic Ovary Syndrome Patients Preconceptionally. Endocr Connect (2019) 8(5):616-24. doi: 10.1530/EC-19-0064

104. Weiss C, Oppelt P, Mayer RB. MATERNAL-FETAL MEDICINE The Participation Rate of Migrant Women in Gestational Diabetes Screening in Austria: A Retrospective Analysis of 3293 Births. Arch Gynecol Obstet (2019) 299:345-51. doi: 10.1007/s00404-018-4964-5

105. Benhalima K, Robyns K, Van Crombrugge P, Deprez N, Seynhave B, Devlieger R, et al. The Effectiveness of Financial Incentives for Smoking Cessation During Pregnancy: Is it From Being Paid or From the Extra Aid? BMC Pregnancy Childbirth (2012) 12:24. doi: 10.1186/1471-2393-12-24

106. De Munck N, Belva F, Van De Velde H, Verheyen G, Stoop D. Closed Oocyte Vitrification and Storage in an Oocyte Donation Programme: Obstetric and Neonatal Outcome. Hum Reprod (2016) 31(5):1024-33. doi: 10.1093/ humrep/dew029

107. Grunewald D, Lahoreau D, Burgel PR, Chapron J, Honore I, Kanaan R, et al. Dietary Management of Pregnant Women With Cystic Fibrosis. J Cystic Fibrosis (2014) 13:S101. doi: 10.1016/S1569-1993(14)60348-9

108. Goire Miailhe G, Kayem G, Girard G, Lè Ne Legardeur H, Mandelbrot L. Selective Rather Than Universal Screening for Gestational Diabetes Mellitus? §. Eur J Obstet Gynecol (2015) 191:95-100. doi: 10.1016/j.ejogrb.2015.05.003

109. Goueslard K, Cottenet J, Mariet A-S, Giroud M, Cottin Y, Petit J-M, et al. Early Cardiovascular Events in Women With a History of Gestational Diabetes Mellitus. Cardiovasc Diabetol (2016) 15:15. doi: 10.1186/s12933016-0338-0

110. Regnault N, Moutengou E, Fosse-Edorh S, Barry Y, Billionnet C, Weill A, et al. Gestational Diabetes Mellitus, Screening Practices, and Association With Social Deprivation: Data From the 788,494 Women Who Delivered in France in 2013. Diabetes (2016) 65:A359. doi: 10.2337/db16-861-1374

111. Mortier I, Blanc J, Tosello B, Gire C, Bretelle F, Carcopino X. Is Gestational Diabetes an Independent Risk Factor of Neonatal Severe Respiratory Distress Syndrome After 34 Weeks of Gestation? A Prospective Study. Arch Gynecol Obstet (2017) 296(6):1071-7. doi: 10.1007/s00404-017-4505-7

112. Boudet-Berquier J, Salanave B, Desenclos J-C, Castetbon K. Sociodemographic Factors and Pregnancy Outcomes Associated With Prepregnancy Obesity: Effect Modification of Parity in the Nationwide Epifane Birth-Cohort. BMC Pregnancy Childbirth (2017) 17(1):1-17. doi: 10.1186/s12884-017-1456-8

113. Billionnet C, Mitanchez D, Weill A, Nizard J, Alla F, Hartemann A, et al. Gestational Diabetes and Adverse Perinatal Outcomes From 716,152 Births in France in 2012. Diabetologia (2017) 60(4):636-44. doi: 10.1007/s00125017-4206-6

114. Mitanchez D, Jacqueminet S, Nizard J, Tanguy ML, Ciangura C, Lacorte JM, et al. Effect of Maternal Obesity on Birthweight and Neonatal Fat Mass: A Prospective Clinical Trial. PloS One (2017) 12(7):1-15. doi: 10.1371/ journal.pone. 0181307

115. Marie C, Léger S, Guttmann A, Rivière O, Marchiset N, Lémery D, et al. Exposure to Arsenic in Tap Water and Gestational Diabetes: A French SemiEcological Study. Environ Res (2018) 161:248-55. doi: 10.1016/ j.envres.2017.11.016
116. Preaubert L, Vincent-Rohfritsch A, Santulli P, Gayet V, Goffinet F, Le Ray C. Outcomes of Pregnancies Achieved by Double Gamete Donation: A Comparison With Pregnancies Obtained by Oocyte Donation Alone. Eur J Obstet Gynecol Reprod Biol (2018) 222:1-6. doi: 10.1016/j.ejogrb.2017.12.026

117. Soomro MH, Baiz N, Huel G, Yazbeck C, Botton J, Heude B, et al. Exposure to Heavy Metals During Pregnancy Related to Gestational Diabetes Mellitus in Diabetes-Free Mothers. Sci Total Environ (2019) 656:870-6. doi: 10.1016/ j.scitotenv.2018.11.422

118. Stuber T, Kunzel E, Zollner U, Rehn M, Wockel A, Honig A. Prevalence and Associated Risk Factors for Obesity During Pregnancy Over Time. GeFra Sci (2015) 75:923-8. doi: 10.1055/s-0035-1557868.pdf

119. Beyerlein A, Koller D, Ziegler AG, Lack N, Maier W. Does Charge-Free Screening Improve Detection of Gestational Diabetes in Women From Deprived Areas: A Cross-Sectional Study. BMC Pregnancy Childbirth (2016) 16(1):1-6. doi: 10.1186/s12884-016-1060-3

120. Tamayo T, Tamayo M, Rathmann W, Potthoff P. Prevalence of Gestational Diabetes and Risk of Complications Before and After Initiation of a General Systematic Two-Step Screening Strategy in Germany (2012-2014). Diabetes Res Clin Pract (2016) 115:1-8. doi: 10.1016/j.diabres.2016.03.001

121. Melchior H, Kurch-Bek D, Mund M. Prävalenz Des Gestationsdiabetes: Eine Populationsbasierte Leistungsdatenanalyse Des Screenings in Der Ambulanten Versorgung. Dtsch Arztebl Int (2017) 114(24):412-8. doi: 10.3238/arztebl.2017.0412

122. Köninger A, Mathan A, Mach P, Frank M, Schmidt B, Schleussner E, et al. Is Afamin a Novel Biomarker for Gestational Diabetes Mellitus? A Pilot Study. Reprod Biol Endocrinol (2018) 16(1):1-11. doi: 10.1186/s12958-018-0338-x

123. Pahlitzsch TMJ, Hanne L, Henrich W, Weichert A. Influence of Foetal Macrosomia on the Neonatal and Maternal Birth Outcome. Geburtshilfe Frauenheilkd (2019) 79(11):1191-8. doi: 10.1055/a-0880-6182

124. Lamain-de Ruiter M, Kwee A, Naaktgeboren CA, de Groot I, Evers IM, Groenendaal F, et al. External Validation of Prognostic Models to Predict Risk of Gestational Diabetes Mellitus in One Dutch Cohort: Prospective Multicentre Cohort Study. BMJ (2016) 354:i4338. doi: 10.1136/bmj.i4338

125. Koning SH, van Zanden JJ, Hoogenberg K, Lutgers HL, Klomp AW, Korteweg FJ, et al. New Diagnostic Criteria for Gestational Diabetes Mellitus and Their Impact on the Number of Diagnoses and Pregnancy Outcomes. Diabetologia (2018) 61(4):800-9. doi: 10.1007/s00125-017-4506-x

126. De Wilde MA, Lamain-De Ruiter M, Veltman-Verhulst SM, Kwee A, Laven JS, Lambalk CB, et al. Increased Rates of Complications in Singleton Pregnancies of Women Previously Diagnosed With Polycystic Ovary Syndrome Predominantly in the Hyperandrogenic Phenotype. Fertil Steril Oे (2017) 108:333-73. doi: 10.1016/j.fertnstert.2017.06.015

127. Mosimann B, Amylidi S, Risch L, Wiedemann U, Surbek D, Baumann M, et al. First-Trimester Placental Growth Factor in Screening for Gestational Diabetes. Fetal Diagn Ther (2016) 39(4):287-91. doi: 10.1159/000441027

128. Amylidi S, Mosimann B, Stettler C, Fiedler GM, Surbek D, Raio L. FirstTrimester Glycosylated Hemoglobin in Women at High Risk for Gestational Diabetes. Acta Obstet Gynecol Scand (2016) 95(1):93-7. doi: 10.1111/ aogs. 12784

129. Ryser Ruetschi J, Jornayvaz F, Rivest R, Huhn E, Irion O, Boulvain M. Fasting Glycaemia to Simplify Screening for Gestational Diabetes. BJOG Int $J$ Obstet Gynaecol (2016) 123(13):2223. doi: 10.1111/1471-0528.13876

130. Horsch A, Kang JS, Vial Y, Ehlert U, Borghini A, Marques-Vidal P, et al. Stress Exposure and Psychological Stress Responses Are Related to Glucose Concentrations During Pregnancy. Br J Health Psychol (2016) 21(3):712-29. doi: $10.1111 /$ bjhp.12197

131. Savopol H, Fontana E, Richli M, Magnin JL, Ben Ali N, Ducry N, et al. Abstracts of 52nd EASD Annual Meeting. Diabetologia (2016) 59(S1):1-581.

132. Djaković I, Sabolović Rudman S, Gall V, Košec A, Markuš Sandrić M, Košec V. Do Changing Diagnostic Criteria For Gestational Diabetes Influence Pregnancy Outcome? Acta Clin Croat (2016) 55(3):422. doi: 10.20471/ acc.2016.55.03.11

133. Djelmis J, Pavić M, Mulliqi Kotori V, Pavlić Renar I, Ivanisevic M, Oreskovic S. Prevalence of Gestational Diabetes Mellitus According to IADPSG and NICE Criteria. Int J Gynecol Obstet (2016) 135(3):250-4. doi: 10.1016/ j.ijgo.2016.07.005

134. Erjavec K, Poljičanin T, Matijević R. Impact of the Implementation of New WHO Diagnostic Criteria for Gestational Diabetes Mellitus on Prevalence 
and Perinatal Outcomes: A Population-Based Study. J Pregnancy (2016) 2016:1-7. doi: 10.1155/2016/2670912

135. Abdulle A, Alnaeemi A, Aljunaibi A, Al Ali A, Al Saedi K, Al Zaabi E, et al. The UAE Healthy Future Study: a Pilot for a Prospective Cohort Study of 20,000 United Arab Emirates Nationals. BMC Public Health. (2018) 18:101. doi: 10.1186/s12889-017-5012-2

136. Inancli IS, Yayci E, Atacag T, Uncu M. Is Maternal Vitamin D Associated With Gestational Diabetes Mellitus in Pregnant Women in Cyprus? Clin Exp Obstet Gynecol (2016) 43(6):840-3. doi: 10.12891/ceog3152.2016

137. Vassilaki M, Chatzi L, Georgiou V, Philalithis A, Kritsotakis G, Koutis A, et al. Pregestational Excess Weight, Maternal Obstetric Complications and Mode of Delivery in the Rhea Cohort in Crete. Eur J Public Health (2015) 25 (4):632-7. doi: 10.1093/eurpub/cku246

138. Trotta F, Da Cas R, Spila S, Gramegna M, Venegoni M, Zocchetti C, et al. Evaluation of Safety of A/H1N1 Pandemic Vaccination During Pregnancy: Cohort Study. BMJ (2014) 348:1-11. doi: 10.1136/bmj.g3361

139. Pintaudi B, Di Vieste G, Corrado F, Lucisano G, Pellegrini F, Giunta L, et al. Improvement of Selective Screening Strategy for Gestational Diabetes Through a More Accurate Definition of High-Risk Groups. Eur J Endocrinol (2014) 170(1):87-93. doi: 10.1530/EJE-13-0759

140. Caserta D, Bordi G, Stegagno M, Filippini F, Podagrosi M, Roselli D, et al. Maternal and Perinatal Outcomes in Spontaneous Versus Assisted Conception Twin Pregnancies. Eur J Obstet Gynecol Reprod Biol (2014) 174(1):64-9. doi: 10.1016/j.ejogrb.2013.12.011

141. Lacaria E, Lencioni C, Russo L, Romano M, Lemmi P, Battini L, et al. Selective Screening for GDM in Italy: Application and Effectiveness of National Guidelines. J Matern Neonatal Med (2015) 28(15):1842-4. doi: 10.3109/14767058.2014.969701

142. D'Anna R, Di Benedetto A, Scilipoti A, Santamaria A, Interdonato ML, Petrella E, et al. Myo-Inositol Supplementation for Prevention of Gestational Diabetes in Obese Pregnant Women: A Randomized Controlled Trial. Obstet Gynecol (2015) 126(2):310-5. doi: 10.1097/AOG.0000000000000958

143. Pinzauti S, Ferrata C, Vannuccini S, Rienzo G, Severi FM, Petraglia F, et al. Twin Pregnancies After Assisted Reproductive Technologies: The Role of Maternal Age on Pregnancy Outcome. Eur J Obstet Gynecol (2016) 206:198203. doi: 10.1016/j.ejogrb.2016.09.019

144. Capula C, Chiefari E, Borelli M, Oliverio R, Vero A, Foti D, et al. A New Predictive Tool for the Early Risk Assessment of Gestational Diabetes Mellitus. Prim Care Diabetes (2016) 10(5):315-23. doi: 10.1016/j.pcd.2016.05.004

145. Santamaria A, Di Benedetto A, Petrella E, Pintaudi B, Corrado F, D’Anna R, et al. Myo-Inositol may Prevent Gestational Diabetes Onset in Overweight Women: A Randomized, Controlled Trial. J Matern Neonatal Med (2016) 29 (19):3234-7. doi: 10.3109/14767058.2015.1121478

146. Bianchi C, Romano M, Aragona M, Battini L, Di Filippi M, Del Prato S, et al. Abstracts of 52nd EASD Annual Meeting. Diabetologia (2016) 59:1-581. doi: 10.1007/s00125-016-4046-9

147. Di Cianni G, Gualdani E, Berni C, Meucci A, Roti L, Lencioni C, et al. Screening for Gestational Diabetes in Tuscany, Italy. A Population Study. Diabetes Res Clin Pract (2017) 132:149-56. doi: 10.1016/j.diabres.2017.08.003

148. Bordi G, D'Ambrosio A, Gallotta I, Di Benedetto L, Frega A, Torcia F, et al. The Influence of Ovulation Induction and Assisted Conception on Maternal and Perinatal Outcomes of Twin Pregnancies. Eur Rev Med Pharmacol Sci (2017) 21(18):3998-4006.

149. Chiefari E, Pastore I, Puccio L, Caroleo P, Oliverio R, Vero A, et al. Impact of Seasonality on Gestational Diabetes Mellitus. Endocrine Metab Immune Disord Drug Targets (2017) 17(3):246-52. doi: 10.2174/1871530317666 170808155526

150. Cozzolino M, Serena C, Maggio L, Rambaldi MP, Simeone S, Mello G, et al. Analysis of the Main Risk Factors for Gestational Diabetes Diagnosed With International Association of Diabetes and Pregnancy Study Groups (IADPSG) Criteria in Multiple Pregnancies. J Endocrinol Invest (2017) 40 (9):937-43. doi: 10.1007/s40618-017-0646-6

151. Bruno R, Petrella E, Bertarini V, Pedrielli G, Neri I, Facchinetti F, et al. Adherence to a Lifestyle Programme in Overweight/Obese Pregnant Women and Effect on Gestational Diabetes Mellitus: a Randomized Controlled Trial. Matern Child Nutr (2017) 13(3).

152. Bianchi C, de Gennaro G, Romano M, Battini L, Aragona M, Corfini M, et al. Italian National Guidelines for the Screening of Gestational Diabetes: Time for a Critical Appraisal? Nutr Metab Cardiovasc Dis (2017) 27(8):717-22. doi: 10.1016/j.numecd.2017.06.010

153. Meregaglia M, Dainelli L, Banks H, Benedetto C, Detzel P, Fattore G. The Short-Term Economic Burden of Gestational Diabetes Mellitus in Italy. BMC Pregnancy Childbirth (2018) 18(1):1-9. doi: 10.1186/s12884-018-1689-1

154. Quaresima P, Visconti F, Chiefari E, Puccio L, Foti DP, Venturella R, et al. Barriers to Postpartum Glucose Intolerance Screening in an Italian Population. Int J Environ Res Public Health (2018) 15(12):1-7. doi: 10.3390/ijerph15122853

155. Gerli S, Favilli A, Franchini D, De Giorgi M, Casucci P, Parazzini F. Is the Robson's Classification System Burdened by Obstetric Pathologies, Maternal Characteristics and Assistential Levels in Comparing Hospitals Cesarean Rates? A Regional Analysis of Class 1 and 3. J Matern Neonatal Med (2018) 31(2):173-7. doi: 10.1080/14767058.2017.1279142

156. Masturzo B, Franzè V, Germano C, Attini R, Gennarelli G, Lezo A, et al. Risk of Adverse Pregnancy Outcomes by Pre-Pregnancy Body Mass Index Among Italian Population: A Retrospective Population-Based Cohort Study on 27,807 Deliveries. Arch Gynecol Obstet (2019) 299(4):983-91. doi: 10.1007/ s00404-019-05093-0

157. Visconti F, Quaresima P, Chiefari E, Caroleo P, Arcidiacono B, Puccio L, et al. First Trimester Combined Test (FTCT) as a Predictor of Gestational Diabetes Mellitus. Int J Environ Res Public Health (2019) 16(19):1-10. doi: 10.3390/ijerph16193654

158. Marozio L, Picardo E, Filippini C, Mainolfi E, Berchialla P, Cavallo F, et al. Maternal Age Over 40 Years and Pregnancy Outcome: A Hospital-Based Survey. J Matern Neonatal Med (2019) 32(10):1602-8. doi: 10.1080/ 14767058.2017.1410793

159. Xuereb S, Magri CJ, Xuereb RA, Xuereb RG, Galea J, Fava S. Gestational Glycemic Parameters and Future Cardiometabolic Risk at Medium-Term Follow Up. Can J Diabetes (2019) 43(8):621-6. doi: 10.1016/ j.jcjd.2019.03.007

160. Kek T, Kuželički NK, Raščan IM, Geršak K. Characteristics of Health Behaviours and Health Status Indicators Among Pregnant Women in Slovenia. Zdr Vestn (2017) 86:295-317. doi: 10.6016/ZdravVestn.2490

161. Goñi L, Cuervo M, Santiago S, Zazpe I, Martínez JA. Influencia De La Paridad Sobre Variables Antropométricas, Estilos De Vida Y Hábitos Alimentarios En Mujeres Embarazadas. Sist Sanit Navar (2014) 37(3):34962. doi: 10.4321/S1137-66272014000300005

162. Ruiz-Gracia T, Duran A, Fuentes M, Rubio MA, Runkle I, Carrera EF, et al. Lifestyle Patterns in Early Pregnancy Linked to Gestational Diabetes Mellitus Diagnoses When Using IADPSG Criteria. The St Carlos Gestational Study. Clin Nutr (2016) 35(3):699-705. doi: 10.1016/j.clnu.2015.04.017

163. Berglund SK, Torres-Espínola FJ, García-Valdés L, Segura MT, MartínezZaldivar C, Padilla C, et al. The Impacts of Maternal Iron Deficiency and Being Overweight During Pregnancy on Neurodevelopment of the Offspring. Br J Nutr (2017) 118(7):533-40. doi: 10.1017/S0007114517002410

164. Benaiges D, Flores-Le Roux JA, Marcelo I, Mañé L, Rodríguez M, Navarro X, et al. Is First-Trimester HbAlc Useful in the Diagnosis of Gestational Diabetes? Diabetes Res Clin Pract (2017) 133:85-91. doi: 10.1016/ j.diabres.2017.08.019

165. Assaf-Balut C, García de la Torre N, Durán A, Fuentes M, Bordiú E, Del Valle L, et al. A Mediterranean Diet With Additional Extra Virgin Olive Oil and Pistachios Reduces the Incidence of Gestational Diabetes Mellitus (GDM): A Randomized Controlled Trial: The St. Carlos GDM Prevention Study. PloS One (2017) 12(10):1-16. doi: 10.1371/journal.pone.0185873

166. Gortazar L, Flores-Le Roux JA, Benaiges D, Sarsanedas E, Payà A, Mañé L, et al. Trends in Prevalence of Gestational Diabetes and Perinatal Outcomes in Catalonia, Spain, 2006 to 2015: The Diagestcat Study. Diabetes Metab Res $\operatorname{Rev}$ (2019) 35(5):e3151. doi: 10.1002/dmrr.3151

167. Mañé L, Flores-Le Roux JA, Benaiges D, Chillarón JJ, Prados M, Pedro-Botet $\mathrm{J}$, et al. Impact of Overt Diabetes Diagnosed in Pregnancy in a Multi-Ethnic Cohort in Spain. Gynecol Endocrinol (2019) 35(4):332-6. doi: 10.1080/ 09513590.2018.1521387

168. International Diabetes Federation. Europe Diabetes Report 2010 - 2045 (2020). Available at: https://diabetesatlas.org/data/en/region/3/eur.html.

169. McIntyre HD, Catalano P, Zhang C, Desoye G, Mathiesen ER, Damm P. Gestational Diabetes Mellitus. Nat Rev Dis Primers (2019) 5:1-19. doi: 10.1038/s41572-019-0098-8 
170. Buckley BS, Harreiter J, Damm P, Corcoy R, Chico A, Simmons D, et al. Gestational Diabetes Mellitus in Europe: Prevalence, Current Screening Practice and Barriers to Screening. A Review. Diabetes Med (2012) 29 (7):844-54. doi: 10.1111/j.1464-5491.2011.03541.x

171. Aroda VR, Christophi CA, Edelstein SL, Zhang P, Herman WH, Barrett-Connor E, et al. The Effect of Lifestyle Intervention and Metformin on Preventing or Delaying Diabetes Among Women With and Without Gestational Diabetes: The Diabetes Prevention Program Outcomes Study 10-Year Follow-Up. J Clin Endocrinol Metab (2015) 100(4):1646-53. doi: 10.1210/jc.2014-3761

Conflict of Interest: The authors declare that the research was conducted in the absence of any commercial or financial relationships that could be construed as a potential conflict of interest.
Publisher's Note: All claims expressed in this article are solely those of the authors and do not necessarily represent those of their affiliated organizations, or those of the publisher, the editors and the reviewers. Any product that may be evaluated in this article, or claim that may be made by its manufacturer, is not guaranteed or endorsed by the publisher.

Copyright (c) 2021 Paulo, Abdo, Bettencourt-Silva and Al-Rifai. This is an openaccess article distributed under the terms of the Creative Commons Attribution License (CC BY). The use, distribution or reproduction in other forums is permitted, provided the original author(s) and the copyright owner(s) are credited and that the original publication in this journal is cited, in accordance with accepted academic practice. No use, distribution or reproduction is permitted which does not comply with these terms. 